\title{
Exact renormalization group flow equations for non-relativistic fermions: scaling towards the Fermi surface
}

\author{
Peter Kopietz and Tom Busche \\ Institut für Theoretische Physik, Universität Frankfurt, Robert-Mayer-Strasse 8, 60054 Frankfurt, Germany
}

(March 30, 2001)

\begin{abstract}
We construct exact functional renormalization group ( $R G$ ) flow equations for non-relativistic fermions in arbitrary dimensions, taking into account not only mode elimination but also the rescaling of the momenta, frequencies and the fermionic fields. The complete RG flow of all relevant, marginal and irrelevant couplings can be described by a system of coupled flow equations for the irreducible $n$-point vertices. Introducing suitable dimensionless variables, we obtain flow equations for generalized scaling functions which are continuous functions of the flow parameter, even if we consider quantities which are dominated by momenta close to the Fermi surface, such as the densitydensity correlation function at long wavelengths. We also show how the problem of constructing the renormalized Fermi surface can be reduced to the problem of finding the RG fixed point of the irreducible two-point vertex at vanishing momentum and frequency. We argue that only if the degrees of freedom are properly rescaled it is possible to reach scale-invariant non-Fermi liquid fixed points within a truncation of the exact RG flow equations.
\end{abstract}

PACS numbers: 71.10-w, 71.10.Hf

\section{INTRODUCTION}

Recently several authors [1] have used exact functional renormalization group (RG) methods to gain a deeper understanding of strongly correlated non-relativistic fermions in reduced dimensions. The exact functional RG yields an infinite hierarchy of coupled differential equations describing the change of the correlation functions due to the elimination and the rescaling of the degrees of freedom. For classical statistical mechanics problems the exact functional RG has been developed long time ago in a pioneering work by Wegner and Houghton [6], who performed the usual three RG-steps [7] 8$]$ to derive their exact flow equation:

1. Integrate out fields with momenta $\mathbf{k}$ in a shell $\Lambda_{0}(1-d t)<|\mathbf{k}|<\Lambda_{0}$, where $d t$ is infinitesimal and $\Lambda_{0}$ is some ultraviolet cutoff.

2. Rescale the remaining momenta by a factor $1+d t$ and express all quantities in terms of the rescaled momenta.

3. Rescale the remaining fields by a factor of $1+\frac{1}{2}(1-\eta) d t$, where the anomalous dimension $\eta$ has to be chosen such that the RG has a fixed point [9].

Over the years several alternative formulations of the exact functional RG in field theory and statistical physics have been proposed 10 15]. Note, however, that recent applications of the exact RG for two-dimensional fermions [1 5. take only the mode elimination step 1 into account, and do not perform the above rescaling steps 2 and 3 . While such a procedure is legitimate if one is interested in solving a given many-body problem iteratively, the Wilsonian RG amounts to more than that [8]: by combining the mode elimination with a suitable rescaling of the degrees of freedom, the Wilsonian RG generates a mapping of the original many-body problem onto a continuous family of new many-body problems, labeled by a flow parameter $t$. In the limit $t \rightarrow \infty$ it sometimes happens that the resulting problem simplifies and can be solved in a controlled manner. In order to obtain a non-trivial RG fixed point for $t \rightarrow \infty$ which is characterized by a non-zero anomalous dimension $\eta$, it is crucial that the fields are properly rescaled, as discussed in detail by Bell and Wilson [9]. For example, by applying the exact functional RG to $\phi^{4}$-theory [13, 16], it is easy to see that without the rescaling the RG flow does not reproduce the Wilson-Fisher fixed point below four dimensions, see Sec.IVC. We therefore believe that the RG calculations of Refs. [1] 5 are incomplete, and should be augmented by the usual rescaling steps 2 and 3 given above. Note that the rescaling is explicitly included in the one loop momentum-shell approach advanced by Shankar [17], so that one may wonder why the exact functional RG used in Refs. [1 5 does not include the rescaling. We shall try to clarify this point in this work by showing how to include the rescaling of the degrees of freedom in the exact functional RG for fermionic many-body systems in arbitrary dimensions. We then point out several advantages of working with the rescaled version of the exact RG flow equations. 


\section{MODE ELIMINATION FOR THE LEGENDRE EFFECTIVE ACTION}

In this section we present a brief derivation of the exact flow equation describing the change in the Legendre effective action $\Gamma\{\bar{\varphi}, \varphi\}$ due to the elimination of the degrees of freedom with momenta and frequencies in a suitably defined shell. Keeping in mind that the Legendre effective action is the generating functional of the one-particle irreducible correlation functions [18], an expansion of $\Gamma\{\bar{\varphi}, \varphi\}$ in powers of the fields $\bar{\varphi}$ and $\varphi$ yields the corresponding flow equations for the irreducible $n$-point vertices. For classical field theories, the flow equation for the Legendre effective action has been considered some time ago by Nicoll et al. [10], and more recently by Wetterich [12], and by Morris [13. For non-relativistic fermions the exact flow equation for the Legendre effective action has recently been derived in a very general form by Honerkamp, Salmhofer and co-authors [4,5], who also included the possibility of symmetry breaking. Our derivation given below is less general but more explicit; in particular, we shall adopt as much as possible the standard notations from the theory of critical phenomena [6, 16].

\section{A. Assumptions and initial conditions}

We consider a translationally invariant non-relativistic system of interacting fermions with a Fermi surface. Throughout this work we assume that the fermions are in the normal state, so that the propagator does not have any anomalous components. By Fermi surface we mean the true Fermi surface of the interacting system, which is defined as the set of all momenta satisfying [19]

$$
\epsilon_{\mathbf{k}_{F}}=\mu-\Sigma\left(\mathbf{k}_{F}, i 0\right)
$$

where $\epsilon_{\mathbf{k}}$ is the energy dispersion of the non-interacting system, $\mu$ is the exact chemical potential and $\Sigma\left(\mathbf{k}, i \omega_{n}\right)$ is the exact self-energy of the interacting many-body system. Here $\mathbf{k}$ is the momentum and $i \omega_{n}$ is the Matsubara frequency. Given an arbitrary $\mathbf{k}$, we may define a corresponding momentum $\mathbf{k}_{F}$ on the Fermi surface via the decomposition 20

$$
\mathbf{k}=\mathbf{k}_{F}+\hat{\mathbf{v}}_{F} p
$$

as shown in Fig.11. Here $\hat{\mathbf{v}}_{F}=\mathbf{v}_{F} /\left|\mathbf{v}_{F}\right|$ is a unit vector in the direction of the local Fermi velocity

$$
\mathbf{v}_{F}=\left.\nabla_{\mathbf{k}} \epsilon_{\mathbf{k}}\right|_{\mathbf{k}=\mathbf{k}_{F}}
$$

Note that $\mathbf{v}_{F}$ is defined in terms of the gradient of the bare energy dispersion at the renormalized Fermi surface. The solution of Eq.(2.1) can be parameterized as

$$
\mathbf{k}_{F}=\hat{\mathbf{n}} k_{F}(\hat{\mathbf{n}})
$$

where $\hat{\mathbf{n}}$ is a unit vector in the direction of $\mathbf{k}_{F}$, see Fig.1. Note that only for a spherical Fermi surface we may identify $\hat{\mathbf{n}}=\hat{\mathbf{v}}_{F}$, so that $\mathbf{k}_{F}$ and $\mathbf{v}_{F}$ are parallel. In this case we know that $\mathbf{k}_{F}$ is not renormalized as we turn on the interactions at constant density [19,21]. The true Fermi surface is then given by $\epsilon_{\mathbf{k}_{F}}=\mu_{0}$ (where $\mu_{0}$ is the chemical potential of the non-interacting system at the same density), so that $\Sigma\left(\mathbf{k}_{F}, i 0\right)=\mu-\mu_{0}$, which is independent of $\mathbf{k}_{F}$.

For the derivation of the RG flow equations it is convenient to represent the generating functionals of the fermionic correlation functions in terms of Grassmannian functional integrals [22]. We assume that initially the Grassmann fields $\psi_{K}$ with large excitation energies $\epsilon_{\mathbf{k}}-\epsilon_{\mathbf{k}_{F}}$ and large Matsubara frequencies $\omega_{n}$ have been integrated out. Here $K=\left(\mathbf{k}, i \omega_{n}\right)$ is a composite label, and for simplicity we ignore the spin degree of freedom. The high energy shell can be described by an equation of the form $\Omega_{K} \gtrsim \xi_{0}$, where $\Omega_{K}$ is a suitable homogeneous function of $\epsilon_{\mathbf{k}}-\epsilon_{\mathbf{k}_{F}}$ or $\left|\omega_{n}\right|$. Possible choices are $\Omega_{K}=\left|\omega_{n}\right|, \Omega_{K}=\left|\epsilon_{\mathbf{k}}-\epsilon_{\mathbf{k}_{F}}\right|$, or $\Omega_{K}=\sqrt{\omega_{n}^{2}+\left(\epsilon_{\mathbf{k}}-\epsilon_{\mathbf{k}_{F}}\right)^{2}}$. Our starting point is an effective action of the form

$$
S_{\xi_{0}}\{\bar{\psi}, \psi\}=S_{\xi_{0}}^{0}\{\bar{\psi}, \psi\}+S_{\xi_{0}}^{\operatorname{int}}\{\bar{\psi}, \psi\}
$$

where the free part is given by

$$
S_{\xi_{0}}^{0}\{\bar{\psi}, \psi\}=\int_{K} \Theta_{\gamma}\left(\xi_{0}-\Omega_{K}\right)\left[-i \omega_{n}+\epsilon_{\mathbf{k}}-\epsilon_{\mathbf{k}_{F}}\right] \bar{\psi}_{K} \psi_{K} .
$$

Here we use the notation 


$$
\int_{K}=\frac{1}{\beta V} \sum_{\mathbf{k}, \omega_{n}} \rightarrow \int \frac{d \mathbf{k}}{(2 \pi)^{D}} \int_{-\infty}^{\infty} \frac{d \omega}{2 \pi},
$$

where $\beta$ is the inverse temperature and $V$ is the volume, and the right-hand side is valid for $\beta \rightarrow \infty$ and $V \rightarrow \infty$. The interaction part is

$$
\begin{aligned}
& S_{\xi_{0}}^{\operatorname{int}}\{\bar{\psi}, \psi\}= \\
& \quad \int_{K} \Theta_{\gamma}\left(\xi_{0}-\Omega_{K}\right)\left[\Sigma_{\xi_{0}}(K)-\Sigma\left(\mathbf{k}_{F}, i 0\right)\right] \bar{\psi}_{K} \psi_{K} \\
& +\frac{1}{(2 !)^{2}} \int_{K_{1}^{\prime}} \int_{K_{2}^{\prime}} \int_{K_{2}} \int_{K_{1}} \delta_{K_{1}^{\prime}+K_{2}^{\prime}, K_{2}+K_{1}} \\
& \quad \times \Gamma_{\xi_{0}}^{(4)}\left(K_{1}^{\prime}, K_{2}^{\prime} ; K_{2}, K_{1}\right) \bar{\psi}_{K_{1}^{\prime}} \bar{\psi}_{K_{2}^{\prime}} \psi_{K_{2}} \psi_{K_{1}}+\ldots,
\end{aligned}
$$

where the ellipsis denotes three-body and higher order interactions, and

$$
\begin{aligned}
\delta_{K K^{\prime}} & =\beta V \delta_{\mathbf{k k}^{\prime}} \delta_{\omega_{n} \omega_{n^{\prime}}} \\
& \rightarrow(2 \pi)^{D+1} \delta\left(\mathbf{k}-\mathbf{k}^{\prime}\right) \delta\left(\omega-\omega^{\prime}\right) .
\end{aligned}
$$

All vertices are antisymmetric with respect to the permutation of any pair of the incoming particles and any pair of the outgoing particles. In particular, the four-point vertex $\Gamma_{\xi}^{(4)}\left(K_{1}^{\prime}, K_{2}^{\prime} ; K_{2}, K_{1}\right)$ is antisymmetric with respect to the exchange $K_{1}^{\prime} \leftrightarrow K_{2}^{\prime}$ and $K_{1} \leftrightarrow K_{2}$. The function $\Theta_{\gamma}(\epsilon)$ is a smooth cutoff function, satisfying $\Theta_{\gamma}(\epsilon) \approx 1$ for $\epsilon>\gamma$ and $\Theta_{\gamma}(\epsilon) \approx 0$ for $\epsilon<-\gamma$, so that $\lim _{\gamma \rightarrow 0} \Theta_{\gamma}(\epsilon)=\Theta(\epsilon)$. The term $\Sigma_{\xi_{0}}(K)$ in Eq. 2.8) is the contribution from the high energy fields with $\Omega_{K} \gtrsim \xi_{0}$ to the irreducible self-energy, and $-\Sigma\left(\mathbf{k}_{F}, i 0\right)$ is a counterterm which takes into account that in the free action (2.6) we have subtracted $\epsilon_{\mathbf{k}_{F}}=\mu-\Sigma\left(\mathbf{k}_{F}, i 0\right)$ from the bare energy dispersion. As shown below, this subtraction is crucial to obtain the RG flow of the Fermi surface. In a perturbative approach, the above subtraction is necessary to obtain a well-behaved perturbation series [19,23]. The counterterm $-\Sigma\left(\mathbf{k}_{F}, i 0\right)$ can be determined from a self-consistency condition, which can be imposed order by order in perturbation theory [24]. Within a RG approach, the counterterm can be determined a posteriori from the condition that the flow equation of the momentum- and frequency-independent part of the irreducible two-point vertex has a fixed point [17], see Sec.IV B for a careful discussion. The energy scale $\xi_{0}$ plays a dual role [13]: For the calculation of the vertices of the initial action $S_{\xi_{0}}\{\bar{\psi}, \psi\}$ the scale $\xi_{0}$ acts as an infrared cutoff, so that for sufficiently large $\xi_{0}$ the vertices appearing in $S_{\xi_{0}}\{\bar{\psi}, \psi\}$ can be calculated perturbatively. On the other hand, for the remaining low-energy degrees of freedom $\xi_{0}$ plays the role of an ultraviolet cutoff.

\section{B. Exact flow equation describing mode elimination}

Starting from the effective action $S_{\xi_{0}}\{\bar{\psi}, \psi\}$ with cutoff $\xi_{0}$, we eliminate all fields with momenta and Matsubara frequencies in the regime $\xi \lesssim \Omega_{K} \lesssim \xi_{0}$. The free propagator for the fermionic fields in this regime has in the $K$-basis the diagonal elements

$$
G_{\xi, \xi_{0}}^{0}(K)=\frac{\Theta_{\gamma}\left(\Omega_{K}-\xi\right)-\Theta_{\gamma}\left(\Omega_{K}-\xi_{0}\right)}{i \omega_{n}-\epsilon_{\mathbf{k}}+\epsilon_{\mathbf{k}_{F}}}
$$

Because $\mathbf{k}_{F}$ lies by construction on the true Fermi surface of the interacting system, the right-hand side of Eq. (2.10) depends implicitly on the interaction. Note, however, that $\epsilon_{\mathbf{k}_{F}}$ is independent of the flow parameter $\xi$. Diagrammatically, the elimination of the degrees of freedom in the shell $\xi \lesssim \Omega_{K} \lesssim \xi_{0}$ corresponds to contracting all terms generated by expanding $e^{-S_{\xi_{0}}^{\text {int }}\{\bar{\psi}, \psi\}}$ with the propagators $G_{\xi, \xi_{0}}^{0}$ given in Eq. $(2.10)$. The connected correlation functions of the new theory can be formally represented as functional derivatives of the generating functional $\mathcal{G}_{\xi}^{c}\{\bar{J}, J\}$ defined by [25]

$$
\begin{aligned}
& e^{\mathcal{G}_{\xi}^{c}\{\bar{J}, J\}}=e^{-S_{\xi_{0}}^{\mathrm{int}}\left\{\zeta \frac{\delta}{\delta J}, \frac{\delta}{\delta J}\right\}} e^{\left(\bar{J},\left(-G_{\xi, \xi_{0}}^{0}\right) J\right)} \\
& =e^{\left(\bar{J},\left(-G_{\xi, \xi_{0}}^{0}\right) J\right)}\left[e^{\left(\zeta \frac{\delta}{\delta \psi},\left(-G_{\xi, \xi_{0}}^{0} \frac{\delta}{\delta \psi}\right)\right.} e^{-S_{\xi_{0}}^{\mathrm{int}}\{\bar{\psi}, \psi\}}\right]_{\psi=G_{\xi, \xi_{0}}^{0} J} .
\end{aligned}
$$

Here $(a, M b)=\int_{K} \int_{K^{\prime}} a_{K} M_{K K^{\prime}} b_{K^{\prime}}$, and the factor $\zeta=-1$ arises from the antisymmetry of the Grassmann fields 22,26]. $G_{\xi, \xi_{0}}^{0}$ should be considered as a matrix in $K$-space, with matrix elements given by 


$$
\left[G_{\xi, \xi_{0}}^{0}\right]_{K K^{\prime}}=\delta_{K K^{\prime}} G_{\xi, \xi_{0}}^{0}(K),
$$

where $G_{\xi, \xi_{0}}^{0}(K)$ is defined in Eq.(2.10).

Differentiating both sides of Eq.(2.11) with respect to $\xi$, we obtain an exact flow equation for the generating functional of the connected correlation functions. For practical calculations the flow equation for the irreducible vertices is more convenient [10,12, 13]. To obtain the corresponding generating functional, we perform a Legendre transformation,

$$
\mathcal{L}_{\xi}\{\bar{\varphi}, \varphi\}=(\bar{\varphi}, J)+(\bar{J}, \varphi)-\mathcal{G}_{\xi}^{c}\{\bar{J}, J\},
$$

where the Grassmann sources $J$ and $\bar{J}$ have to be considered as functionals of the Grassmann fields $\varphi$ and $\bar{\varphi}$ by solving the following equations for $\bar{J}=\bar{J}\{\bar{\varphi}, \varphi\}$ and $J=J\{\bar{\varphi}, \varphi\}$,

$$
\varphi=\zeta \frac{\delta \mathcal{G}_{\xi}^{c}\{\bar{J}, J\}}{\delta J}, \bar{\varphi}=\frac{\delta \mathcal{G}_{\xi}^{c}\{\bar{J}, J\}}{\delta \bar{J}} .
$$

The generating functional of the irreducible vertices is then given by

$$
\Gamma_{\xi}\{\bar{\varphi}, \varphi\}=\mathcal{L}_{\xi}\{\bar{\varphi}, \varphi\}-\left(\bar{\varphi},\left[-G_{\xi, \xi_{0}}^{0}\right]^{-1} \varphi\right),
$$

and satisfies the exact flow equation [4.5.5.27]

$$
\begin{aligned}
\partial_{\xi} \Gamma_{\xi}= & \zeta \int_{K} \partial_{\xi}\left[G_{\xi, \xi_{0}}^{0}(K)\right]^{-1} \\
\times & \left\{\hat{G}_{\xi, \xi_{0}} \hat{\mathcal{U}}_{\xi}\left[\hat{1}-\hat{G}_{\xi, \xi_{0}} \hat{\mathcal{U}}_{\xi}\right]^{-1} \hat{G}_{\xi, \xi_{0}}\right. \\
& \left.+\hat{G}_{\xi, \xi_{0}}^{0} \hat{\Sigma}_{\xi}\left[1-\hat{G}_{\xi, \xi_{0}}^{0} \hat{\Sigma}_{\xi}\right]^{-1} \hat{G}_{\xi, \xi_{0}}^{0}\right\}_{22 ; K K},
\end{aligned}
$$

with initial condition

$$
\Gamma_{\xi_{0}}\{\bar{\varphi}, \varphi\}=S_{\xi_{0}}^{\operatorname{int}}\{\bar{\varphi}, \varphi\} .
$$

Here $\{\ldots\}_{22 ; K K}$ denotes the lower diagonal element of the corresponding $2 \times 2$-matrix in $K$-space, and the functional $\hat{\mathcal{U}}_{\xi}$ is given by

$$
\left(\begin{array}{cc}
\zeta \frac{\delta^{2} \Gamma_{\xi}}{\delta \bar{\varphi}_{K} \delta \varphi_{K^{\prime}}} & \zeta \frac{\delta^{2} \Gamma_{\xi}}{\delta \bar{\varphi}_{K} \delta \bar{\varphi}_{K^{\prime}}} \\
\frac{\delta^{2} \Gamma_{\xi}}{\delta \varphi_{K} \delta \varphi_{K^{\prime}}} & \frac{\delta^{2} \Gamma_{\xi}}{\delta \varphi_{K} \delta \bar{\varphi}_{K^{\prime}}}
\end{array}\right)=\left[\hat{\Sigma}_{\xi}\right]_{K K^{\prime}}+\left[\hat{\mathcal{U}}_{\xi}\{\bar{\varphi}, \varphi\}\right]_{K K^{\prime}},
$$

where $\hat{\Sigma}_{\xi}$ is defined as the field-independent part of the second functional derivative of $\Gamma_{\xi}$, so that $\hat{\mathcal{U}}_{\xi}\{0,0\}=0$. The interacting cutoff-regularized propagator is related to the non-interacting one via the Dyson equation,

$$
\hat{G}_{\xi, \xi_{0}}=\left[\hat{G}_{\xi, \xi_{0}}^{0}-\hat{\Sigma}_{\xi}\right]^{-1},\left[\hat{G}_{\xi, \xi_{0}}^{0}\right]_{i j}=\delta_{i j} G_{\xi, \xi_{0}}^{0} .
$$

\section{Flow equation for sharp cutoff}

For simplicity we shall from now on work with a sharp cutoff function, $\Theta(\epsilon)=\lim _{\gamma \rightarrow 0} \Theta_{\gamma}(\epsilon)$. Due to translational invariance the $K$-dependence of all vertices is constrained by energy-momentum conservation, so that the matrix elements of $\hat{\Sigma}_{\xi}$ are

$$
\left[\hat{\Sigma}_{\xi}\right]_{i j ; K K^{\prime}}=\delta_{i j} \delta_{K K^{\prime}}\left[\Sigma_{\xi}(K)-\Sigma\left(\mathbf{k}_{F}, i 0\right)\right],
$$

where the term $\Sigma\left(\mathbf{k}_{F}, i 0\right)$ is due to the subtraction in Eq.(2.8). The exact flow equation (2.16) can then be reduced to the following form [13, 16 ] 


$$
\begin{aligned}
& \partial_{\xi} \Gamma_{\xi}= \\
& \quad \zeta \int_{K} \frac{\delta\left(\Omega_{K}-\xi\right)}{i \omega_{n}-\epsilon_{\mathbf{k}}+\mu-\Sigma_{\xi}(K)}\left\{\hat{\mathcal{U}}_{\xi}\left[\hat{1}-\hat{G}_{\xi, \xi_{0}} \hat{\mathcal{U}}_{\xi}\right]^{-1}\right\}_{22 ; K K} \\
& \quad-\zeta \beta V \int_{K} \delta\left(\Omega_{K}-\xi\right) \ln \left[\frac{i \omega_{n}-\epsilon_{\mathbf{k}}+\mu-\Sigma_{\xi}(K)}{i \omega_{n}-\epsilon_{\mathbf{k}}+\epsilon_{\mathbf{k}_{F}}}\right] .
\end{aligned}
$$

We now expand

$$
\begin{aligned}
\Gamma_{\xi}\{\bar{\varphi}, \varphi\} & =\sum_{n=0}^{\infty} \frac{(-1)^{n}}{(n !)^{2}} \int_{K_{1}^{\prime}} \ldots \int_{K_{n}^{\prime}} \int_{K_{n}} \ldots \int_{K_{1}} \\
& \times \delta_{K_{1}^{\prime}+\ldots+K_{n}^{\prime}, K_{n}+\ldots+K_{1}} \\
& \times \Gamma_{\xi}^{(2 n)}\left(K_{1}^{\prime}, \ldots, K_{n}^{\prime} ; K_{n}, \ldots, K_{1}\right) \\
& \times \bar{\varphi}_{K_{1}^{\prime}} \cdots \bar{\varphi}_{K_{n}^{\prime}} \varphi_{K_{n}} \cdots \varphi_{K_{1}},
\end{aligned}
$$

and identify the terms with the same powers of the fields on both sides of Eq.(2.21). Note that in the normal state only the even vertices are non-zero. In this way we obtain the RG flow equations for the unrescaled irreducible $2 n$-point vertices $\Gamma_{\xi}^{(2 n)}\left(K_{1}^{\prime}, \ldots, K_{n}^{\prime} ; K_{n}, \ldots, K_{1}\right)$. We now explicitly give the exact flow equations for the vertices $\Gamma_{\xi}^{(0)}, \Gamma_{\xi}^{(2)}$,

and $\Gamma_{\xi}^{(4)}$. For a two-loop calculation one needs the flow equation for the six-point vertex $\Gamma_{\xi}^{(6)}$, which is given in the Appendix.

\section{Free energy}

The interaction correction to the free energy is obtained from the last term in Eq.(2.21),

$$
\partial_{\xi} \Gamma_{\xi}^{(0)}=-\zeta \beta V \int_{K} \delta\left(\Omega_{K}-\xi\right) \ln \left[\frac{i \omega_{n}-\epsilon_{\mathbf{k}}+\mu-\Sigma_{\xi}(K)}{i \omega_{n}-\epsilon_{\mathbf{k}}+\epsilon_{\mathbf{k}_{F}}}\right] .
$$

\section{Self-energy}

Comparing the terms quadratic in the fields on both sides of Eq.(2.21) and using the fact that by construction the two-point vertex is related to the subtracted irreducible self-energy via

$$
\Gamma_{\xi}^{(2)}(K ; K)=-\left[\Sigma_{\xi}(K)-\Sigma\left(\mathbf{k}_{F}, i 0\right)\right]
$$

we obtain

$$
\begin{aligned}
& \partial_{\xi} \Gamma_{\xi}^{(2)}(K ; K)=-\partial_{\xi} \Sigma_{\xi}(K)= \\
& -\zeta \int_{K^{\prime}} \frac{\delta\left(\Omega_{K^{\prime}}-\xi\right)}{i \omega_{n^{\prime}}-\epsilon_{\mathbf{k}^{\prime}}+\mu-\Sigma_{\xi}\left(K^{\prime}\right)} \Gamma_{\xi}^{(4)}\left(K, K^{\prime} ; K^{\prime}, K\right) .
\end{aligned}
$$

A graphical representation of this equation is shown in Fig.2.

\section{Four-point vertex}

The flow equation for the irreducible four-point vertex is 


$$
\begin{aligned}
& \partial_{\xi} \Gamma_{\xi}^{(4)}\left(K_{1}^{\prime}, K_{2}^{\prime} ; K_{2}, K_{1}\right)= \\
& -\zeta \int_{K} \frac{\delta\left(\Omega_{K}-\xi\right)}{i \omega_{n}-\epsilon_{\mathbf{k}}+\mu-\Sigma_{\xi}(K)} \Gamma_{\xi}^{(6)}\left(K_{1}^{\prime}, K_{2}^{\prime}, K ; K, K_{2}, K_{1}\right) \\
& +\int_{K}\left[\frac{\delta\left(\Omega_{K}-\xi\right) G_{\xi, \xi_{0}}\left(K^{\prime}\right)}{i \omega_{n}-\epsilon_{\mathbf{k}}+\mu-\Sigma_{\xi}(K)}+\frac{G_{\xi, \xi_{0}}(K) \delta\left(\Omega_{K^{\prime}}-\xi\right)}{i \omega_{n^{\prime}}-\epsilon_{\mathbf{k}^{\prime}}+\mu-\Sigma_{\xi}\left(K^{\prime}\right)}\right] \\
& \quad \times\left\{\frac{1}{2}\left[\Gamma_{\xi}^{(4)}\left(K_{1}^{\prime}, K_{2}^{\prime} ; K^{\prime}, K\right) \Gamma_{\xi}^{(4)}\left(K, K^{\prime}, K_{2}, K_{1}\right)\right]_{K^{\prime}=K_{1}+K_{2}-K}\right. \\
& \quad+\zeta\left[\Gamma_{\xi}^{(4)}\left(K_{1}^{\prime}, K^{\prime} ; K, K_{1}\right) \Gamma_{\xi}^{(4)}\left(K_{2}^{\prime}, K ; K^{\prime}, K_{2}\right)\right]_{K^{\prime}=K+K_{1}-K_{1}^{\prime}} \\
& \left.\quad+\left[\Gamma_{\xi}^{(4)}\left(K_{2}^{\prime}, K^{\prime} ; K, K_{1}\right) \Gamma_{\xi}^{(4)}\left(K_{1}^{\prime}, K ; K^{\prime}, K_{2}\right)\right]_{K^{\prime}=K+K_{1}-K_{2}^{\prime}}\right\} .
\end{aligned}
$$

This equation is shown graphically in Fig. 3. The first term in the curly braces is contribution from the BCS-channel, while the last two terms are the zero-sound contributions, usually abbreviated by ZS (second term) and $\mathrm{ZS}^{\prime}$ (third term) [17. Note that both zero-sound terms have to be retained in order to preserve the antisymmetry of the four-point vertex [28]. The exact flow equation for the six-point vertex is rather lengthy and is given in the Appendix.

\section{EXACT FLOW EQUATIONS DESCRIBING MODE ELIMINATION AND RESCALING}

So far we have derived exact flow equations for the irreducible vertices describing the elimination of the degrees of freedom. Within the one-loop approximation, it is sufficient to set $\Gamma_{\xi}^{(2 n)}=0$ for $n \geq 3$, and to ignore interaction corrections to the propagators in internal loops. The resulting truncated flow equation for the four-point vertex in two dimensions has been analyzed numerically by Honerkamp et al. [4,5]. A similar numerical analysis of an equivalent truncated flow equation has been performed by Halboth and Metzner [2,3]. Both groups found that the one-loop flow of the four-point vertex eventually diverges at a finite scale, where the perturbative RG breaks down. Physically the runaway flow has been interpreted in terms of some incipient instability of the normal metallic phase. Here we would like to point out that in principle there is another interpretation of this runaway flow, namely the existence of a scale-invariant non-Fermi liquid fixed point, which is characterized by a finite anomalous dimension. Below we argue that the mode elimination RG transformations of Refs. [1 5 cannot detect such a fixed point, because these equations do not take into account that the degrees of freedom should be properly rescaled to reach a fixed point with a finite anomalous dimension [9]. Assuming the existence of such a fixed point, only the rescaled version of the exact flow equations given below would detect it, while the pure mode elimination RG used in Refs. [1 [5] would still exhibit a runaway flow to strong coupling.

Because the following rescaling procedure depends crucially on the existence of a Fermi surface, we now explicitly set the statistics factor $\zeta=-1$. We also take the limits of infinite volume $(V \rightarrow \infty)$ and zero temperature $(\beta \rightarrow \infty)$, so that momenta and frequencies become continuous variables.

\section{A. Scaling variables}

Instead of the momentum $\mathbf{k}$ and the Matsubara frequency $i \omega$, we now label the degrees of freedom by the direction $\hat{\mathbf{n}}$ of $\mathbf{k}_{F}$ (see Fig.11) and by the dimensionless variables

$$
q=\frac{v_{F} p}{\xi}=\frac{\mathbf{v}_{F} \cdot\left(\mathbf{k}-\mathbf{k}_{F}\right)}{\xi}, \epsilon=\frac{\omega}{\xi}
$$

so that

$$
\mathbf{k}=\mathbf{k}_{F}+\hat{\mathbf{v}}_{F} \frac{\xi}{v_{F}} q=\hat{\mathbf{n}} k_{F}(\hat{\mathbf{n}})+\hat{\mathbf{v}}_{F} \frac{\xi}{v_{F}} q .
$$

Furthermore, instead of the flow parameter $\xi$, we introduce the dimensionless logarithmic flow parameter

$$
t=-\ln \left(\xi / \xi_{0}\right)
$$

and define the dimensionless dispersion 


$$
\xi_{t}^{\hat{\mathbf{n}}}(q)=\frac{\epsilon_{\mathbf{k}}-\epsilon_{\mathbf{k}_{F}}}{\xi}=q+\frac{c_{t}^{\hat{\mathbf{n}}}}{2} q^{2}+O\left(q^{3}\right),
$$

where

$$
c_{t}^{\hat{\mathbf{n}}}=\frac{\xi}{m v_{F}^{2}}=\frac{\xi_{0}}{m v_{F}^{2}} e^{-t}
$$

is an irrelevant coupling which measures the leading deviation from linearity in the energy dispersion in the direction normal to the Fermi surface. In Eq.(3.4) we have expanded the energy dispersion around $\mathbf{k}=\mathbf{k}_{F}$,

$$
\epsilon_{\mathbf{k}}=\epsilon_{\mathbf{k}_{F}}+\mathbf{v}_{F} \cdot\left(\mathbf{k}-\mathbf{k}_{F}\right)+\frac{\left(\mathbf{k}-\mathbf{k}_{F}\right)^{2}}{2 m}+\ldots
$$

Note that in general $\mathbf{v}_{F}$ and $m$ depend on $\hat{\mathbf{n}}$. Using $Q=(\hat{\mathbf{n}}, q, i \epsilon)$ instead of $K=(\mathbf{k}, i \omega)$ as integration variables, we have for $\beta \rightarrow \infty$ and $V \rightarrow \infty$

$$
\int_{K}=\nu_{0} \xi^{2} \int_{Q}=\nu_{0} \xi^{2} \int \frac{d S_{\hat{\mathbf{n}}}}{S_{D}} \int d q J(\hat{\mathbf{n}}, q) \int \frac{d \epsilon}{2 \pi},
$$

where $d S_{\hat{\mathbf{n}}}$ is a surface element and $S_{D}$ is the surface area of the unit sphere in $D$ dimensions, and $J(\hat{\mathbf{n}}, q)$ is a dimensionless Jacobian associated with the transformation $\mathbf{k} \rightarrow(\hat{\mathbf{n}}, q)$. For convenience we have pulled out a factor of $\nu_{0} \xi^{2}$ in Eq.(3.7), where $\nu_{0}$ is the density of states at the Fermi surface,

$$
\nu_{0}=\int \frac{d \mathbf{k}}{(2 \pi)^{D}} \delta\left(\epsilon_{\mathbf{k}}-\epsilon_{\mathbf{k}_{F}}\right) .
$$

With this normalization $J(\hat{\mathbf{n}}, q)$ is dimensionless. In particular, for a spherical Fermi surface

$$
\nu_{0}=\frac{\Omega_{D}}{(2 \pi)^{D}} \frac{k_{F}^{D-1}}{v_{F}} \quad, J(\hat{\mathbf{n}}, q)=\left(1+c_{t} q\right)^{D-1} .
$$

\section{B. The scaling form of the irreducible vertices}

The proper definition of the dimensionless scaling form of the irreducible vertices follows partially from dimensional analysis, and partially from aesthetic considerations (such as the requirement that numerical prefactors should be as simple as possible). Given the expansion (2.22) of the generating functional of the irreducible vertices in powers of the dimensionful fields $\varphi_{K}$, we substitute

$$
\varphi_{K}=\left(\frac{Z_{t}^{\hat{\mathbf{n}}}}{\xi^{3} \nu_{0}}\right)^{1 / 2} \tilde{\varphi}_{Q}
$$

where the wave-function renormalization factor $Z_{t}^{\hat{\mathbf{n}}}$ is related to the irreducible self-energy $\Sigma_{\xi}(\mathbf{k}, i \omega)$ at scale $\xi$ as usual,

$$
Z_{t}^{\hat{\mathbf{n}}}=\frac{1}{1-\left.\frac{\partial \Sigma_{\xi}\left(\mathbf{k}_{F}, i \omega\right)}{\partial(i \omega)}\right|_{\omega=0}}
$$

The dimensionless fields $\tilde{\varphi}_{Q}$ should be considered as functions of the scaling variables $Q$.

\section{Free energy}

Due to the Jacobian associated with the rescaling of fermionic fields in the functional integral, the field-independent part $\Gamma_{\xi}^{(0)}$ of the generating functional $\Gamma_{\xi}\{\bar{\varphi}, \varphi\}$ picks up an additive term, so that after rescaling the correction to the free energy is

$$
\tilde{\Gamma}_{t}^{(0)}=\Gamma_{\xi}^{(0)}+\beta V \int_{K} \Theta\left(\xi-\Omega_{K}\right) \ln Z_{t}^{\hat{\mathbf{n}}} .
$$

In classical statistical mechanics a contribution analogous to the second term has been discussed by Wegner and Houghton [6]. 


\section{Two-point vertex}

We write the exact propagator in the following scaling form,

$$
G_{\xi, \xi_{0}}(\mathbf{k}, i \omega)=\frac{Z_{t}^{\hat{\mathbf{n}}}}{\xi} \tilde{G}_{t}\left(\frac{\mathbf{k}_{F}}{k_{F}}, \frac{\mathbf{v}_{F} \cdot\left(\mathbf{k}-\mathbf{k}_{F}\right)}{\xi}, \frac{i \omega}{\xi}\right),
$$

where $\hat{\mathbf{n}}$ should be considered as a function of $\mathbf{k}$, as given in Eqs.(2.2) and (2.4). Note that our exact RG equations describe the flow of the dimensionless scaling function

$$
\tilde{G}_{t}(Q) \equiv \tilde{G}_{t}(\hat{\mathbf{n}}, q, i \epsilon)=\frac{\xi}{Z_{t}^{\hat{\mathbf{n}}}} G_{\xi, \xi_{0}}\left(\hat{\mathbf{n}} k_{F}(\hat{\mathbf{n}})+\hat{\mathbf{v}}_{F} \frac{\xi}{v_{F}} q, i \xi \epsilon\right)
$$

Introducing the dimensionless scaling form of the (subtracted) irreducible two-point vertex,

$$
\tilde{\Gamma}_{t}^{(2)}(Q)=\frac{Z_{t}^{\hat{\mathbf{n}}}}{\xi} \Gamma_{\xi}^{(2)}(K)=-\frac{Z_{t}^{\hat{\mathbf{n}}}}{\xi}\left[\Sigma_{\xi}(K)-\Sigma\left(\mathbf{k}_{F}, i 0\right)\right]
$$

and the scaling form of the inverse propagator

$$
\begin{aligned}
r_{t}(Q) & =\frac{Z_{t}^{\hat{\mathbf{n}}}}{\xi}\left[i \omega-\epsilon_{\mathbf{k}}+\mu-\Sigma_{\xi}(\mathbf{k}, i \omega)\right] \\
& =Z_{t}^{\hat{\mathbf{n}}}\left[i \epsilon-\xi_{t}^{\hat{\mathbf{n}}}(q)\right]+\tilde{\Gamma}_{t}^{(2)}(Q)
\end{aligned}
$$

we obtain

$$
\begin{aligned}
\tilde{G}_{t}(Q) & =\frac{\Theta\left(\tilde{\Omega}_{Q}-1\right)-\Theta\left(\tilde{\Omega}_{Q}-e^{t}\right)}{r_{t}(Q)} \\
& =\frac{\Theta\left(e^{t}>\tilde{\Omega}_{Q}>1\right)}{r_{t}(Q)}
\end{aligned}
$$

where

$$
\Theta\left(x_{2}>x>x_{1}\right)=\left\{\begin{array}{cc}
1 & \text { if } x_{2}>x>x_{1} \\
0 & \text { else }
\end{array}\right.
$$

and

$$
\tilde{\Omega}_{Q}=\frac{\Omega_{K}}{\xi}
$$

Note that for the choice $\Omega_{K}=\left|\epsilon_{\mathbf{k}}-\epsilon_{\mathbf{k}_{F}}\right|$ we obtain $\tilde{\Omega}_{Q}=\left|\xi_{t}^{\hat{\mathbf{n}}}(q)\right| \approx|q|$ to leading order. The initial condition at $t=0$ implies

$$
\begin{aligned}
r_{0}(Q) & =\frac{Z_{0}^{\hat{\mathbf{n}}}}{\xi_{0}}\left[i \omega-\epsilon_{\mathbf{k}}+\mu-\Sigma_{\xi_{0}}(K)\right] \\
& =Z_{0}^{\hat{\mathbf{n}}}\left[i \epsilon-\xi_{0}^{\hat{\mathbf{n}}}(q)\right]+\tilde{\Gamma}_{0}^{(2)}(Q) .
\end{aligned}
$$

Furthermore, without interactions $Z_{t}=1$ and $\tilde{\Gamma}_{t}^{(2)}(Q)=0$, so that $r_{t}(Q)=i \epsilon-\xi_{t}^{\hat{\mathbf{n}}}(q)$.

\section{Higher order vertices $(n \geq 2)$}

The dimensionless scaling form of the higher order irreducible vertices follows from our definition (3.10) of the dimensionless fields,

$$
\begin{aligned}
\tilde{\Gamma}_{t}^{(2 n)}( & \left.Q_{1}^{\prime}, \ldots, Q_{n}^{\prime} ; Q_{n}, \ldots, Q_{1}\right)= \\
& \nu_{0}^{n-1} \xi^{n-2}\left[Z_{t}^{\hat{\mathbf{n}}_{1}^{\prime}} \ldots Z_{t}^{\hat{\mathbf{n}}_{n}^{\prime}} Z_{t}^{\hat{\mathbf{n}}_{n}} \ldots Z_{t}^{\hat{\mathbf{n}}_{1}}\right]^{1 / 2} \\
& \times \Gamma_{\xi}^{(2 n)}\left(K_{1}^{\prime}, \ldots, K_{n}^{\prime} ; K_{n}, \ldots, K_{1}\right)
\end{aligned}
$$




\section{Flow equations for the rescaled vertices}

\section{Free energy}

Defining the interaction correction to the free energy per Fourier component,

$$
f_{t}=\frac{\tilde{\Gamma}_{t}^{(0)}}{\beta V \int_{K} \Theta\left(\xi-\Omega_{K}\right)},
$$

and using Eqs.(2.23) and (3.12), we obtain the exact flow equation for $f_{t}$,

$$
\partial_{t} f_{t}=f_{t}-\int \frac{d S_{\hat{\mathbf{n}}}}{S_{D}} \eta_{t}^{\hat{\mathbf{n}}}-\frac{\int_{Q} \delta\left(\tilde{\Omega}_{Q}-1\right) \ln \left(\frac{r_{t}(Q)}{i \epsilon-\xi_{t}^{\hat{n}}(q)}\right)}{\int_{Q} \Theta\left(1-\tilde{\Omega}_{Q}\right)} .
$$

Here $\eta_{t}^{\hat{\mathbf{n}}}$ is the flowing anomalous dimension,

$$
\eta_{t}^{\hat{\mathbf{n}}}=-\partial_{t} \ln Z_{t}^{\hat{\mathbf{n}}}=-\frac{\partial_{t} Z_{t}^{\hat{\mathbf{n}}}}{Z_{t}^{\hat{\hat{n}}}} .
$$

Eq.(3.23) is the fermionic analog of the corresponding flow equation for the free energy in $\phi^{4}$-theory, see Eq.(4.6) of Ref. [16].

\section{Two-point vertex}

From Eqs.(2.25) and (3.15) we find the flow equation for the dimensionless subtracted two-point vertex,

$$
\begin{aligned}
\partial_{t} \tilde{\Gamma}_{t}^{(2)}(Q) & =\left(1-\eta_{t}^{\hat{\mathbf{n}}}-Q \cdot \partial_{Q}\right) \tilde{\Gamma}_{t}^{(2)}(Q) \\
& -\int_{Q^{\prime}} \dot{G}_{t}\left(Q^{\prime}\right) \tilde{\Gamma}_{t}^{(4)}\left(Q, Q^{\prime} ; Q^{\prime}, Q\right),
\end{aligned}
$$

where we have introduced the notation

$$
\begin{aligned}
Q \cdot \partial_{Q} & =q \partial_{q}+\epsilon \partial_{\epsilon}, \\
\dot{G}_{t}(Q) & =\frac{\delta\left(\tilde{\Omega}_{Q}-1\right)}{r_{t}(Q)} .
\end{aligned}
$$

\section{Four-point vertex}

The flow equation for the rescaled irreducible four-point vertex follows from Eqs. 2.26) and (3.21),

$$
\begin{aligned}
& \partial_{t} \tilde{\Gamma}_{t}^{(4)}\left(Q_{1}^{\prime}, Q_{2}^{\prime} ; Q_{2}, Q_{1}\right)= \\
& -\sum_{i=1}^{2}\left[\frac{\eta_{t}^{\mathbf{n}_{i}^{\prime}}+\eta_{t}^{\hat{\mathbf{n}}_{i}}}{2}+Q_{i}^{\prime} \cdot \partial_{Q_{i}^{\prime}}+Q_{i} \cdot \partial_{Q_{i}}\right] \tilde{\Gamma}_{t}^{(4)}\left(Q_{1}^{\prime}, Q_{2}^{\prime} ; Q_{2}, Q_{1}\right) \\
& -\int_{Q} \dot{G}_{t}(Q) \tilde{\Gamma}_{t}^{(6)}\left(Q_{1}^{\prime}, Q_{2}^{\prime}, Q ; Q, Q_{2}, Q_{1}\right) \\
& -\int_{Q}\left[\dot{G}_{t}(Q) \tilde{G}_{t}\left(Q^{\prime}\right)+\tilde{G}_{t}(Q) \dot{G}_{t}\left(Q^{\prime}\right)\right] \\
& \times\left\{\frac{1}{2}\left[\tilde{\Gamma}_{t}^{(4)}\left(Q_{1}^{\prime}, Q_{2}^{\prime} ; Q^{\prime}, Q\right) \tilde{\Gamma}_{t}^{(4)}\left(Q, Q^{\prime} ; Q_{2}, Q_{1}\right)\right]_{K^{\prime}=K_{1}+K_{2}-K}\right. \\
& \quad-\left[\tilde{\Gamma}_{t}^{(4)}\left(Q_{1}^{\prime}, Q^{\prime} ; Q, Q_{1}\right) \tilde{\Gamma}_{t}^{(4)}\left(Q_{2}^{\prime}, Q ; Q^{\prime}, Q_{2}\right)\right]_{K^{\prime}=K+K_{1}-K_{1}^{\prime}} \\
& \left.\quad+\left[\tilde{\Gamma}_{t}^{(4)}\left(Q_{2}^{\prime}, Q^{\prime} ; Q, Q_{1}\right) \tilde{\Gamma}_{t}^{(4)}\left(Q_{1}^{\prime}, Q ; Q^{\prime}, Q_{2}\right)\right]_{K^{\prime}=K+K_{1}-K_{2}^{\prime}}\right\}
\end{aligned}
$$


Here $K, K^{\prime}, K_{i}$ and $K_{i}^{\prime}$ should be considered as functions of the dimensionless scaling variables introduced in Sec. III A, for example $K=\left(\hat{\mathbf{n}} k_{F}+\hat{\mathbf{v}}_{F} \frac{\xi}{v_{F}} q, i \xi \epsilon\right)$. Due to the non-linearity of this transformation, the explicit expression of $Q^{\prime}$ in terms of $Q$ and $Q_{i}=\left(\hat{\mathbf{n}}_{i}, q_{i}, \epsilon_{i}\right)$ is rather complicated. For example, let us calculate $Q^{\prime}=\left(\hat{\mathbf{n}}^{\prime}, q^{\prime}, \epsilon^{\prime}\right)$ in the zero-sound contribution involving $K^{\prime}=K+K_{1}-K_{1}^{\prime}$. The energy component is simple, $\epsilon^{\prime}=\epsilon+\epsilon_{1}-\epsilon_{1}^{\prime}$, but for $\hat{\mathbf{n}}^{\prime}$ and $q^{\prime}$ we obtain

$$
\begin{gathered}
\hat{\mathbf{n}}^{\prime}=\frac{\hat{\mathbf{n}}+\hat{\mathbf{n}}_{1}-\hat{\mathbf{n}}_{1}^{\prime}+c_{t}\left(\hat{\mathbf{n}} q+\hat{\mathbf{n}}_{1} q_{1}-\hat{\mathbf{n}}_{1}^{\prime} q_{1}^{\prime}\right)}{\left|\hat{\mathbf{n}}+\hat{\mathbf{n}}_{1}-\hat{\mathbf{n}}_{1}^{\prime}+c_{t}\left(\hat{\mathbf{n}} q+\hat{\mathbf{n}}_{1} q_{1}-\hat{\mathbf{n}}_{1}^{\prime} q_{1}^{\prime}\right)\right|}, \\
q^{\prime}=\frac{1}{c_{t}}\left[\left|\hat{\mathbf{n}}+\hat{\mathbf{n}}_{1}-\hat{\mathbf{n}}_{1}^{\prime}+c_{t}\left(\hat{\mathbf{n}} q+\hat{\mathbf{n}}_{1} q_{1}-\hat{\mathbf{n}}_{1}^{\prime} q_{1}^{\prime}\right)\right|-1\right],
\end{gathered}
$$

where $c_{t}=\xi_{0} e^{-t} /\left(m v_{F}^{2}\right)$, see Eq.(3.5). For simplicity we have assumed a spherical Fermi surface, so that $\mathbf{k}_{i}=$ $\hat{\mathbf{n}}_{i} k_{F}\left(1+c_{t} q_{i}\right)$. Note that the corresponding rescaled energy dispersion is

$$
\begin{aligned}
\xi_{t}^{\hat{\mathbf{n}}^{\prime}}\left(q^{\prime}\right) & =q+\hat{\mathbf{n}} \cdot\left(\hat{\mathbf{n}}_{1} q_{1}-\hat{\mathbf{n}}_{1}^{\prime} q_{1}^{\prime}\right)+\frac{\hat{\mathbf{n}} \cdot\left(\hat{\mathbf{n}}_{1}-\hat{\mathbf{n}}_{1}^{\prime}\right)}{c_{t}} \\
& +\frac{c_{t}}{2}\left(\hat{\mathbf{n}} q+\hat{\mathbf{n}}_{1} q_{1}-\hat{\mathbf{n}}_{1}^{\prime} q_{1}^{\prime}\right)^{2}+\frac{\left(\hat{\mathbf{n}}_{1}-\hat{\mathbf{n}}_{1}^{\prime}\right)^{2}}{2 c_{t}} \\
& +\left(\hat{\mathbf{n}}_{1}-\hat{\mathbf{n}}_{1}^{\prime}\right) \cdot\left(\hat{\mathbf{n}} q+\hat{\mathbf{n}}_{1} q_{1}-\hat{\mathbf{n}}_{1}^{\prime} q_{1}^{\prime}\right) .
\end{aligned}
$$

The rescaled flow equation for the six-point vertex is given in the Appendix.

\section{ADVANTAGES OF RESCALED FLOW EQUATIONS}

In this section we discuss several properties of the rescaled flow equations of Sec.III, and argue that for practical calculations it may be advantageous to use the rescaled flow equations instead of the unrescaled equations discussed in Sec. [I].

\section{A. Screening and flow of the density-density correlation function within RPA}

As pointed out in Refs. [2 [- [), the unrescaled flow equations predict pathological RG flows for physical quantities which are determined by degrees of freedom in the immediate vicinity of the Fermi surface, such as uniform susceptibilities: for any finite infrared cutoff $\xi$ uniform susceptibilities are not renormalized at all, while at $\xi=0$ their RG flow exhibits a discontinuity. Although for a Fermi liquid one may use the Fermi liquid relations between the uniform susceptibilities and the quasi-particle interactions to obtain flow equations for the susceptibilities at finite cutoff $\xi$ [2.3], it would be better to calculate uniform susceptibilities entirely within the framework of the exact RG, without relying on the assumption that the system is a Fermi liquid.

To illustrate the above point, let us consider the screening of the effective interaction, which is closely related to the density-density correlation function. The screening problem has also been considered by Shankar (see Appendix A of Ref. [17]) within the field theory version of the RG, and by Dupuis [29] within the conventional Kadanoff-Wilson RG scheme for Fermi liquids.

Consider the flow equation $(2.26)$ for the irreducible four-point vertex. Suppose we start from a bare interaction at scale $\xi_{0}$ of the form

$$
\Gamma_{\xi_{0}}^{(4)}\left(K_{1}^{\prime}, K_{2}^{\prime} ; K_{2}, K_{1}\right)=f_{\xi_{0}}\left(K_{1}-K_{1}^{\prime}\right)-f_{\xi_{0}}\left(K_{1}-K_{2}^{\prime}\right) .
$$

Let us now calculate the flow of the effective interaction within the random-phase approximation (RPA), where the BCS-contribution is ignored and only those zero-sound terms are retained which preserve the form (4.1). Setting for simplicity $K_{1}=K_{1}^{\prime}$ and $K_{2}=K_{2}^{\prime}$ (this is the combination appearing in the flow equation (2.25) for the self-energy) we approximate the irreducible four-point vertex at scale $\xi<\xi_{0}$ by

$$
\Gamma_{\xi}^{(4)}\left(K_{1}, K_{2} ; K_{2}, K_{1}\right) \approx-f_{\xi}\left(K_{1}-K_{2}\right),
$$

where we have assumed $f_{\xi}(0)=0$. In this approximation we obtain from Eq. 2.26) 


$$
\partial_{\xi} f_{\xi}^{-1}\left(K_{1}-K_{2}\right)=\dot{\Pi}_{\xi}\left(K_{1}-K_{2}\right)
$$

where the change of the polarization is given by

$$
\begin{gathered}
\dot{\Pi}_{\xi}\left(K_{1}-K_{2}\right)=\int_{K}\left[\frac{\delta\left(\Omega_{K}-\xi\right) G_{\xi, \xi_{0}}\left(K+K_{1}-K_{2}\right)}{i \omega_{n}-\epsilon_{\mathbf{k}}+\mu-\Sigma_{\xi}(K)}\right. \\
\left.+\frac{G_{\xi, \xi_{0}}(K) \delta\left(\Omega_{K+K_{1}-K_{2}}-\xi\right)}{i \omega_{n+n_{1}-n_{2}}-\epsilon_{\mathbf{k}+\mathbf{k}_{1}-\mathbf{k}_{2}}+\mu-\Sigma_{\xi}\left(K+K_{1}-K_{2}\right)}\right] .
\end{gathered}
$$

Within the RPA we ignore the self-energy corrections to the propagators in Eq.(4.4). Using the cutoff function $\Omega_{K}=\left|\epsilon_{\mathbf{k}}-\epsilon_{\mathbf{k}_{F}}\right|$, we obtain for $\left|\mathbf{k}_{1}-\mathbf{k}_{2}\right| \ll k_{F}$

$$
\begin{aligned}
\dot{\Pi}_{\xi}\left(K_{1}-K_{2}\right) & \approx 2 \delta(\xi) \int \frac{d \mathbf{k}}{(2 \pi)^{D}} \delta\left(\epsilon_{\mathbf{k}}-\epsilon_{\mathbf{k}_{F}}\right) \\
& \times \frac{\mathbf{v}_{F} \cdot\left(\mathbf{k}_{1}-\mathbf{k}_{2}\right)}{i\left(\omega_{n_{1}}-\omega_{n_{2}}\right)-\mathbf{v}_{F} \cdot\left(\mathbf{k}_{1}-\mathbf{k}_{2}\right)} .
\end{aligned}
$$

Obviously, $\dot{\Pi}_{\xi}(P)=0$ for any $\xi \neq 0$, so that the RPA interaction $f_{\xi}(P)$ is not renormalized for any finite $\xi$. Due to the factor $\delta(\xi)$ in Eq. 4.5) the RG flow of the RPA interaction $f_{\xi}(P)$ (and hence the flow of the polarization and the compressibility) is discontinuous at $\xi=0$. This discontinuity is smoothed out if one works at a finite temperature [29,30], but there is a way to avoid discontinuous flow equations at zero temperature: the RG flow equations generated by our rescaled version of the exact RG remain continuous even at $T=0$.

We now explicitly show this for the RG-flow of the rescaled density-density correlation function. Within the RPA this the rescaled four-point vertex is approximated by

$$
\tilde{\Gamma}_{t}^{(4)}\left(Q_{1}, Q_{2} ; Q_{2}, Q_{1}\right) \approx-F_{t}\left(Q_{1}, Q_{2}\right),
$$

where the dimensionless function $F_{t}\left(Q_{1}, Q_{2}\right)$ satisfies the flow equation

$$
\left[\partial_{t}-Q_{1} \cdot \partial_{Q_{1}}-Q_{2} \cdot \partial_{Q_{2}}\right] F_{t}^{-1}\left(Q_{1}, Q_{2}\right)=-\dot{\tilde{\Pi}}_{t}\left(Q_{1}, Q_{2}\right),
$$

which is the rescaled analog of Eq. (4.3). Here

$$
\dot{\tilde{\Pi}}_{t}\left(Q_{1}, Q_{2}\right)=\int_{Q}\left[\dot{G}_{t}(Q) \tilde{G}_{t}\left(Q^{\prime}\right)+\tilde{G}_{t}(Q) \dot{G}_{t}\left(Q^{\prime}\right)\right]_{K^{\prime}=K+K_{1}-K_{2}},
$$

where $Q^{\prime}=Q^{\prime}\left(Q, Q_{1}, Q_{2}\right)$ is defined as a function of $Q=(\hat{\mathbf{n}}, q, i \epsilon), Q_{1}=\left(\hat{\mathbf{n}}_{1}, q_{1}, i \epsilon_{1}\right)$ and $Q_{2}=\left(\hat{\mathbf{n}}_{2}, q_{2}, i \epsilon_{2}\right)$ via $K^{\prime}=K+K_{1}-K_{2}$, as explained in Sec.III . Ignoring again interaction corrections to the propagators and working with the momentum shell $\tilde{\Omega}_{Q}=\left|\epsilon_{\mathbf{k}}-\epsilon_{\mathbf{k}_{F}}\right| / \xi=\left|\xi_{t}^{\hat{\mathbf{n}}}(q)\right|$, we have

$$
\begin{gathered}
\tilde{G}_{t}(Q)=\frac{\Theta\left(e^{t}>\left|\xi_{t}^{\hat{\mathbf{n}}}(q)\right|>1\right)}{i \epsilon-\xi_{t}^{\hat{\mathbf{n}}}(q)}, \\
\dot{G}_{t}(Q)=\frac{\delta\left(\left|\xi_{t}^{\hat{\mathbf{n}}}(q)\right|-1\right)}{i \epsilon-\xi_{t}^{\hat{\mathbf{n}}}(q)} .
\end{gathered}
$$

Substituting these expressions into Eq.(4.8) and performing the integrations over $q$ and $\epsilon$ we obtain

$$
\dot{\tilde{\Pi}}_{t}\left(Q_{1}, Q_{2}\right)=-4 \int \frac{d S_{\hat{\mathbf{n}}}}{S_{D}} \frac{\Theta\left(1+e^{t}>\hat{\mathbf{n}} \cdot \mathbf{q}_{12}>2\right) \hat{\mathbf{n}} \cdot \mathbf{q}_{12}}{\left(\hat{\mathbf{n}} \cdot \mathbf{q}_{12}\right)^{2}+\left(\epsilon_{1}-\epsilon_{2}\right)^{2}},
$$

where we have used Eq.(3.31) to simplify $\xi_{t}^{\hat{\mathbf{n}}^{\prime}}\left(q^{\prime}\right)$ for small $\left|\mathbf{k}_{1}-\mathbf{k}_{2}\right|$,

$$
\xi_{t}^{\hat{\mathbf{n}}^{\prime}}\left(q^{\prime}\right) \approx q+\hat{\mathbf{n}} \cdot \mathbf{q}_{12}, \quad \mathbf{q}_{12}=\hat{\mathbf{n}}_{1} q_{1}-\hat{\mathbf{n}}_{2} q_{2}+\frac{\hat{\mathbf{n}}_{1}-\hat{\mathbf{n}}_{2}}{c_{t}} .
$$


The crucial point is now that, unlike Eq.4.5), the right-hand side of Eq.(4.11) is a non-singular function of the flowparameter $t$. In fact, in dimensions $D>1$ the $\Theta$-function discontinuity of the integrand disappears after the angular integration, so that $\dot{\tilde{\Pi}}_{t}\left(Q_{1}, Q_{2}\right)$ is a continuous function of $t$. But even in $D=1$, where the angular integration should be replaced by a summation over the two discrete Fermi points, there is no discontinuity in the solution of the flow equation (4.7).

It is instructive to elaborate a little bit more on the case $D=1$, where we obtain from Eq.4.11)

$$
\dot{\tilde{\Pi}}_{t}\left(Q_{1}, Q_{2}\right)=-2 \delta_{\hat{\mathbf{n}}_{1}, \hat{\mathbf{n}}_{2}} \frac{\left|q_{1}-q_{2}\right| \Theta\left(1+e^{t}>\left|q_{1}-q_{2}\right|>2\right)}{\left(q_{1}-q_{2}\right)^{2}+\left(\epsilon_{1}-\epsilon_{2}\right)^{2}} .
$$

In deriving this expression we have assumed $c_{t}\left|q_{i}\right| \ll 1$, and have neglected the terms with $\hat{\mathbf{n}}_{1}=-\hat{\mathbf{n}}_{2}$, which vanish exponentially for $t \rightarrow \infty$. Note that the right-hand side of Eq.(4.13) has discontinuities, but no $\delta$-function singularity, in contrast to Eq. (4.5). It follows that the flow of the physical density-density correlation function is continuous. To see this, let us explicitly solve Eq.(4.7). Using the method described in Ref. [16], the solution with the correct initial condition is easily obtained,

$$
\begin{aligned}
& F_{t}^{-1}\left(Q_{1}, Q_{2}\right)=F_{t=0}^{-1}\left(Q_{1}, Q_{2}\right) \\
& \quad-\int_{0}^{t} d \tau \dot{\tilde{\Pi}}_{t-\tau}\left(\hat{\mathbf{n}}_{1}, e^{-\tau} q_{1}, e^{-\tau} i \epsilon_{1} ; \hat{\mathbf{n}}_{2}, e^{-\tau} q_{2}, e^{-\tau} i \epsilon_{2}\right) .
\end{aligned}
$$

Substituting Eq.(4.13) into Eq.(4.14) we finally obtain in $D=1$

$$
\begin{aligned}
F_{t}^{-1}\left(Q_{1}, Q_{2}\right) & =F_{t=0}^{-1}\left(Q_{1}, Q_{2}\right) \\
& +\delta_{\hat{\mathbf{n}}_{1}, \hat{\mathbf{n}}_{2}} \tilde{\Pi}_{t}\left(q_{1}-q_{2}, i \epsilon_{1}-i \epsilon_{2}\right),
\end{aligned}
$$

where

$$
\begin{aligned}
\tilde{\Pi}_{t}(q, i \epsilon) & =\frac{|q|}{q^{2}+\epsilon^{2}}\left[(|q|-2) \Theta\left(1+e^{t}>|q|>2\right)\right. \\
& \left.+\left(2 e^{t}-|q|\right) \Theta\left(2 e^{t}>|q|>1+e^{t}\right)\right] .
\end{aligned}
$$

Note that the function $x \Theta(x)$ is continuous, so that the right-hand side of Eq.(4.16) is indeed a continuous function of $t$. The physical density-density correlation function is then

$$
\begin{aligned}
\Pi_{\xi}(p, i \omega) & =\nu_{0} \tilde{\Pi}_{t}\left(\frac{v_{F} p}{\xi}, \frac{i \omega}{\xi}\right) \\
= & \frac{1}{\pi}\left[\frac{|p|\left(v_{F}|p|-2 \xi\right) \Theta\left(\xi+\xi_{0}>v_{F}|p|>2 \xi\right)}{\left(v_{F} p\right)^{2}+\omega^{2}}\right. \\
& \left.+\frac{|p|\left(2 \xi_{0}-v_{F}|p|\right) \Theta\left(2 \xi_{0}>v_{F}|p|>\xi+\xi_{0}\right)}{\left(v_{F} p\right)^{2}+\omega^{2}}\right] .
\end{aligned}
$$

Taking the limits $\xi_{0} \rightarrow \infty$ and $\xi \rightarrow 0$ we obtain the well-known result

$$
\Pi_{0}(p, i \omega)=\frac{v_{F}}{\pi} \frac{p^{2}}{\left(v_{F} p\right)^{2}+\omega^{2}} .
$$

From the first line in Eq. (4.17) it is clear why our rescaled flow equations are more suitable for the calculation of the susceptibilities than the corresponding unrescaled flow equations: Because the dependence of the density-density correlation function on the infrared cutoff appears in the scaling function $\tilde{\Pi}_{t}\left(\frac{v_{F} p}{\xi}, \frac{i \omega}{\xi}\right)$ via the ratios $p / \xi$ and $\omega / \xi$, the result for uniform susceptibilities depends on the order in which the limits $p \rightarrow 0$ and $\xi \rightarrow 0$ are taken. Our rescaled RG equation directly yields the scaling function $\tilde{\Pi}_{t}\left(\frac{v_{F} p}{\xi}, \frac{i \omega}{\xi}\right)$ where this problem does not arise. Let us give an alternative explanation for this difference: in the unrescaled flow equations the momenta and frequencies are held constant as the infrared cutoff is reduced. Any fixed distance from the Fermi surface is therefore magnified on the scale of the reduced infrared cutoff, so that momenta that are initially close to the Fermi surface are mapped onto new momenta which, on the reduced scale, appear further away from the Fermi surface. On the other hand, in the rescaled flow equations the momenta and frequencies are scaled down together with the infrared cutoff, so that the degrees of freedom that are initially in the vicinity of the Fermi surface are mapped onto coarse grained degrees of freedom that remain close to the Fermi surface. 


\section{B. The Fermi surface as a RG fixed point manifold}

As already emphasized in Sec.IIA, to obtain well-behaved scaling properties we should expand the bare energy dispersion around the true Fermi surface of the interacting many-body problem, which a priori is not known. We now show how the shape of the Fermi surface can be calculated a posteriori from the fixed point equation for the momentum- and frequency-independent part of the irreducible two-point vertex,

$$
\tilde{\mu}_{t}^{\hat{\mathbf{n}}}=\tilde{\Gamma}_{t}^{(2)}(\hat{\mathbf{n}}, 0, i 0)
$$

Note that this is a relevant coupling function (labeled by the direction $\hat{\mathbf{n}}$ ) with scaling dimension +1 . There are two marginal coupling functions associated with the two-point vertex. One of them is the usual wave-function renormalization given in Eq.(3.11). In terms of our rescaled two-point vertex it can also be written as

$$
Z_{t}^{\hat{\mathbf{n}}}=1-\left.\frac{\partial \tilde{\Gamma}_{t}^{(2)}(\hat{\mathbf{n}}, q=0, i \epsilon)}{\partial(i \epsilon)}\right|_{\epsilon=0}
$$

The other marginal coupling can be taken to be the dimensionless renormalization factor of the Fermi velocity

$$
\tilde{v}_{t}^{\hat{\mathbf{n}}}=Z_{t}^{\hat{\mathbf{n}}}-\left.\frac{\partial \tilde{\Gamma}_{t}^{(2)}(\hat{\mathbf{n}}, q, i 0)}{\partial q}\right|_{q=0} .
$$

The RG flow of the shape of the Fermi surface of strongly correlated electrons in reduced dimensions have recently been considered by several authors [17,3, 1,31 . However, the interpretation of the renormalized Fermi surface in terms of a RG fixed point manifold has only been emphasized by Shankar [17], who used the conventional one-loop momentum shell technique, including the rescaling step. In contrast, the authors of Ref. [3, 4, 31, work with unrescaled flow equations, in which case the coupling corresponding to Eq.(4.19) does not flow to a RG fixed point, so that the interpretation of the Fermi surface as a RG fixed point manifold is obscured.

To study the shape of the Fermi surface within the exact RG, let us retain only relevant and marginal couplings in the two-point function. In this approximation

$$
r_{t}(Q) \approx i \epsilon-\tilde{v}_{t}^{\hat{\mathbf{n}}} q+\tilde{\mu}_{t}^{\hat{\mathbf{n}}}
$$

It is convenient to define the Fermi surface momenta $\mathbf{k}_{F, t}$ of the system with cutoff $\xi=\xi_{0} e^{-t}$ in analogy with Eq.(2.1),

$$
\epsilon_{\mathbf{k}_{F, t}}=\mu-\Sigma_{\xi}\left(\mathbf{k}_{F, t}, i 0\right)
$$

so that the true renormalized Fermi surface is $\mathbf{k}_{F}=\lim _{t \rightarrow \infty} \mathbf{k}_{F, t}$, see Eq.(2.1). In order to calculate $\mathbf{k}_{F, t}$ we use the fact that by construction $q$ in Eq.(4.22) is measured relative to the renormalized $\mathbf{k}_{F}$, so that we obtain from the definition (4.23), up to irrelevant terms,

$$
\mathbf{k}_{F, t}=\mathbf{k}_{F}+\hat{\mathbf{v}}_{F} \frac{\xi_{0} e^{-t} \tilde{\mu}_{t}^{\hat{\mathbf{n}}}}{v_{F} \tilde{v}_{t}^{\hat{\mathbf{n}}}} .
$$

Setting $t=0$ we obtain for the difference between the fully renormalized Fermi momentum $\mathbf{k}_{F}$ and the Fermi momentum $\mathbf{k}_{F, 0}$ of the model with cutoff $\xi_{0}$,

$$
\mathbf{k}_{F}-\mathbf{k}_{F, 0}=-\hat{\mathbf{v}}_{F} \frac{\xi_{0} \tilde{\mu}_{0}^{\hat{\mathbf{n}}}}{v_{F} \tilde{v}_{0}^{\hat{\mathbf{n}}}} .
$$

It should be kept in mind that $\mathbf{v}_{F}$ is defined in terms of the gradient of the bare energy dispersion at the renormalized $\mathbf{k}_{F}$. From Eqs. 3.15 and (4.19) we find

$$
\xi_{0} \tilde{\mu}_{0}^{\hat{\mathbf{n}}}=Z_{0}^{\hat{\mathbf{n}}}\left[\Sigma\left(\mathbf{k}_{F}, i 0\right)-\Sigma_{\xi_{0}}\left(\mathbf{k}_{F}, i 0\right)\right]
$$

which allows us to reconstruct the counterterm $\Sigma\left(\mathbf{k}_{F}, i 0\right)$ as follows: Suppose that we have adjusted the initial condition $\tilde{\mu}_{0}^{\hat{\mathbf{n}}}$ such that for $t \rightarrow \infty$ the relevant couplings $\tilde{\mu}_{t}^{\hat{\mathbf{n}}}$ approach a fixed point. Due to the relevance of $\tilde{\mu}_{t}^{\hat{\mathbf{n}}}$, we expect that this requires some fine tuning of the initial $\tilde{\mu}_{0}^{\hat{\mathbf{n}}}$, so that on the critical manifold (i.e. the manifold in parameter space that flows into a RG fixed point) $\tilde{\mu}_{0}^{\hat{n}}$ becomes a function of the other relevant and marginal couplings. From Eq.(4.26) we then obtain the counterterm $\Sigma\left(\mathbf{k}_{F}, i 0\right)$ as a function of these couplings. Note that if we adjust the 
initial conditions such that $\tilde{\mu}_{t}^{\hat{\mathbf{n}}}$ remains finite for $t \rightarrow \infty$, then Eq.4.24) guarantees that for $t \rightarrow \infty$ the flowing $\mathbf{k}_{F, t}$ indeed approaches the true Fermi surface. Thus, the problem of constructing the renormalized Fermi surface can be reduced to the problem of finding the $R G$ fixed point of the flow equation for the relevant coupling function $\tilde{\mu}_{t}^{\hat{n}}$.

The above discussion relies on the approximation (4.22) for the inverse propagator, which is only justified for a Fermi liquid. In principle it is also possible that the system flows to a non-Fermi liquid fixed point, characterized by some new renormalized Fermi surface. As pointed out by Anderson [32], in this case there might be some subtleties related to the subtraction of the counterterm $\Sigma\left(\mathbf{k}_{F}, i 0\right)$.

It is also worth emphasizing the following point: In order to reach a RG fixed point, it is crucial that the bare energy dispersion is expanded around the fully renormalized Fermi surface $\mathbf{k}_{F}$, defined in Eq.(2.1). If we had chosen the bare Fermi surface $\mathbf{k}_{F, 0}$ as the reference for the expansion, then the RG flow of the relevant coupling $\tilde{\mu}_{t}^{\hat{\mathbf{n}}}$ would exhibit a runaway flow, i.e. $\left|\tilde{\mu}_{t}^{\hat{n}}\right| \rightarrow \infty$ for $t \rightarrow \infty$. To see this, suppose that we expand around the initial Fermi surface at scale $\xi_{0}$. Then the relation (4.24) between the renormalized and the bare Fermi momentum is replaced by

$$
\mathbf{k}_{F, t}=\mathbf{k}_{F, 0}+\hat{\mathbf{v}}_{F, 0} \frac{\xi_{0} e^{-t} \tilde{\mu}_{t}^{\hat{\mathbf{n}}}}{v_{F, 0} \tilde{v}_{t}^{\hat{\mathbf{n}}}}
$$

where $\mathbf{v}_{F, 0}=\left.\nabla_{\mathbf{k}} \epsilon_{\mathbf{k}}\right|_{\mathbf{k}_{F, 0}}$. Given that $\mathbf{k}_{F, t}$ approaches for large $t$ a value that is different from the bare $\mathbf{k}_{F, 0}$, we see from Eq. (4.27) that $\tilde{\mu}_{t}^{\hat{\mathbf{n}}}$ must necessarily diverge as $e^{t}$ (assuming that $\tilde{v}_{t}^{\hat{\mathbf{n}}}$ remains finite). This runaway flow indicates that we have expanded around the wrong Fermi surface. This phenomenon is well known from the usual theory of critical phenomena, where the fixed point manifold is discrete: In this case a runaway flow indicates the existence of some new fixed point. What is new here is that the fixed point manifold is a continuum, which can therefore continuously change under RG transformations. To avoid a runaway flow, we have to expand all quantities from the beginning around the true Fermi surface, which can be determined a posteriori from the requirement that the RG flow indeed approaches a fixed point.

\section{Rescaling and the possibility of non-Fermi liquid fixed points}

Long time ago Bell and Wilson [9] pointed out that the field rescaling (see step 3 in Sec.I) is necessary in order to obtain a non-trivial RG fixed point, characterized by a non-zero anomalous dimension $\eta$. In fact, the RG fixed point conditions can be viewed as some sort of non-linear eigenvalue equations, which contain $\eta$ as an adjustable parameter [33]. From this point of view it is not surprising [15] that solutions of the fixed point equations exist only for certain values of $\eta$, depending on the interaction, the dimensionality, and the symmetries of the system. We believe that the above statement is also true for fermionic many-body systems, so that the unrescaled flow equations used in Refs. [1] [0 cannot be used to detect non-Fermi liquid fixed points.

Because within a one-loop approximation the anomalous dimension vanishes, at the first sight it seems that at this level of approximation it is sufficient to work with a pure mode-elimination RG [1 15 . This is not necessarily true, however, because even at the one-loop level the rescaling of momenta and frequencies (see step 2 in Sec.il) can be essential to obtain a non-trivial RG fixed point. For example, consider $\phi^{4}$-theory slightly below $D=4$ dimensions. In this case there are two relevant couplings, namely the momentum-independent part of the irreducible two-point vertex $\tilde{\mu}_{t}$, and the momentum-independent part of the irreducible four-point vertex $\tilde{g}_{t}$. The one-loop RG flow equations for suitably defined [16] dimensionless couplings are well known [18]

$$
\begin{aligned}
& \partial_{t} \tilde{\mu}_{t}=2 \tilde{\mu}_{t}+\frac{\tilde{g}_{t}}{2\left(1+\tilde{\mu}_{t}\right)}, \\
& \partial_{t} \tilde{g}_{t}=(4-D) \tilde{g}_{t}-\frac{3 \tilde{g}_{t}^{2}}{2\left(1+\tilde{\mu}_{t}\right)^{2}} .
\end{aligned}
$$

For $0<4-D \ll 1$ these equations have a non-trivial fixed point at

$$
\tilde{g}^{*} \approx \frac{2}{3}(4-D), \quad \tilde{\mu}^{*} \approx-\frac{1}{6}(4-D),
$$

which arises from a balance between the competing effects due to the scaling terms (i.e. the first terms on the righthand sides of Eqs.(4.28) and (4.29)) and the mode elimination terms (i.e. the second terms on the right-hand sides of Eqs.(4.28) and (4.29)). Obviously, a RG transformation which includes only the mode elimination would miss the Wilson-Fisher fixed point. 
Finally, we point out that the rescaling of momenta and frequencies plays also an important role to accelerate the flow of the irrelevant couplings to negligibly small values. For example, the constant part of the rescaled irreducible six-point vertex $\tilde{\Gamma}_{t}^{(6)}\left(Q_{1}^{\prime}, Q_{2}^{\prime}, Q_{3}^{\prime} ; Q_{3}, Q_{2}, Q_{1}\right)$ is irrelevant with scaling dimension -1 . This -1 appears as the first term on the right-hand side of Eq.(A.4), and causes the size of the rescaled six-point vertex to diminish exponentially as we iterate the RG (as long as this decrease is not overwhelmed by some anomalous dimension). On the other hand, the corresponding flow equation (A.1) for the unrescaled six-point vertex does not exhibit a similar suppression due to a negative scaling dimension, so that there is no guarantee that the unrescaled six-point vertex really becomes small as we iterate the RG transformation.

\section{SUMMARY AND CONCLUSIONS}

In this work we have constructed exact functional RG equations for non-relativistic fermions, taking not only the mode elimination into account, but also the rescaling of momenta, frequencies, and fields. The exact flow equations given in this work are valid in arbitrary dimensions and describe the RG flow of any translationally invariant fermionic many-body system in the normal state. We emphasize that these equations are exact and describe the RG flow of the irreducible correlation functions of the many-body system for all momenta and frequencies. Of course, it is possible to extract the usual perturbative RG $\beta$-function describing the flow of the momentum-independent part of the fourpoint vertex from these flow equations. To obtain the $\beta$-function at the two-loop order, it is sufficient to truncate the infinite hierarchy of flow equations by setting $\Gamma^{(2 n)}=0$ for $n \geq 4$. In Sections IIC, III C and in the Appendix we have explicitly written down all expressions necessary for performing a two-loop calculation for any $D$-dimensional translationally invariant normal fermionic many-body system. These equations are also valid in $D=1$, where twoloop calculations are usually performed using the field theory-method [34]. Note, however, that in this case there are some subtleties which are not fully understood [35].

We have formulated the exact functional RG for fermionic systems in close analogy with the functional RG for field theories and statistical mechanics problems. We have tried to emphasize this analogy by adopting as much as possible the notation used in statistical mechanics [6.16]. A crucial step is the introduction of dimensionless scaling variables and rescaled vertices, which describe the scaling towards the true Fermi surface of the system. Finally, in Sec.IV we have shown that the rescaled flow equations for the irreducible vertices given in Sec.IIIC have several advantages as compared with the unrescaled flow equations discussed in Sec.IIC:

1. Flow of susceptibilities. The unrescaled exact RG equations used in Refs. [1 [ [ ] yield discontinuities in flow equations for quantities which are dominated by degrees of freedom in the immediate vicinity of the Fermi surface, such as the density-density correlation function at long wavelengths. In contrast, our formulation of the exact RG including rescaling yields flow equations which are continuous functions of the flow parameter.

2. Renormalization of the shape of the Fermi surface. The calculation of the shape of the renormalized Fermi surface can be reduced to the solution of the fixed point equation for the properly rescaled irreducible two-point vertex at vanishing external momentum and frequency. Without rescaling, the corresponding flow equation does not have a fixed point, so that the associated relevant coupling exhibits a runaway flow.

3. Non-Fermi liquid fixed points. If the system has a scale invariant non-Fermi liquid fixed point characterized by a non-zero anomalous dimension $\eta$, then the flow generated by RG transformations which omit the rescaling steps 2 and 3 listed in Sec. of the type used in Refs. [1 5 ] would generate a runaway-flow to strong coupling in this case.

We suspect that our rescaled flow equations will also turn out to be advantageous for numerical calculations, because they describe the RG flow of the relevant scaling functions. The numerical analysis of these equations is a rather tedious task [2, [- which is beyond the scope of this work.

Our flow equations also offer a new approach to study interacting fermions in $D=1$, where the normal metallic state is known to be a non-Fermi liquid. For these systems the RG $\beta$-function is often calculated within the field theory formulation of the RG advanced by Sólyom [34]. However, till now there exists no RG calculation of the full momentum- and frequency-dependent single-particle Green function of interacting fermions in $D=1$. From bosonization it is known that in momentum-frequency space the single-particle Green function exhibits interesting features such as power-law singularities, which are a manifestation of anomalous scaling and spin-charge-separation [36]. We are currently investigating whether the full spectral function of interacting fermions in $D=1$ can be calculated by means of an approximate solution of the rescaled functional RG equation (3.25) for the two-point vertex. We have preliminary evidence [37] that at weak coupling this can indeed be done, and that for the Tomonaga-Luttinger model the resulting spectral function compares quite well with the exact result obtained via bosonization. 
Finally, we would like to address the question whether possible scale-invariant non-Fermi liquid fixed points in dimensions $D>1$ are accessible within the exact RG. Certainly, detecting such a fixed point requires at least a two-loop calculation, because at the one-loop level the wave-function renormalization $Z_{t}^{\hat{\mathbf{n}}}$ remains unity. Considering the complexity of numerical analysis of the one-loop equations describing the RG flow without the rescaling steps [2] 5], the direct numerical analysis of the two-loop RG flow equations including rescaling seems to be a rather difficult task. Such a calculation would require the numerical analysis of the flow equation for the six-point vertex given in Eq. (A.4). Note, however, that in the special case of a square Fermi surface Binz, Baeriswyl and Douçot [38] have recently presented an analytic study of the one-loop RG flow in the vicinity of the dominant instabilities. Let us emphasize again that $Z_{t}^{\hat{\mathbf{n}}} \rightarrow 0$ at a non-Fermi liquid fixed point, so that the inclusion of the flow of the wave-function renormalization is crucial to reach such a fixed point.

For Fermi surfaces with a special geometry it is possible to analyze the RG flow analytically [38, 39]. In particular, very recently Ferraz 39] performed a field-theoretic two-loop RG calculation for a two-dimensional Fermi system with a truncated Fermi surface, consisting of flat and curved pieces. He succeeded to calculate the entire single-particle spectral function. Interestingly, he found non-Fermi liquid behavior for all points on the Fermi surface, including the curved pieces. The calculations presented in Ref. [39] are quite encouraging and support our point of view that higher-dimensional non-Fermi liquid fixed points are accessible within a two-loop truncation of the Wilsonian RG equations presented in this work.

\section{ACKNOWLEDGMENTS}

We thank B. Binz, A. Ferraz, C. Honerkamp, V. Meden, M. Salmhofer, and K. Schönhammer for discussions, and N. Dupuis for pointing out some relevant references. This work was financially supported by the DFG-Schwerpunkt SSP 1053, project No.SCHO201/8-1.

\section{APPENDIX: FLOW EQUATIONS FOR THE IRREDUCIBLE SIX-POINT VERTEX}

For a two-loop calculation we need the flow equation for the irreducible six-point vertex [13,16, 37]. The unrescaled flow equation reads (see Fig. 1 and recall that for fermions $\zeta=-1$ )

$$
\begin{aligned}
& \partial_{\xi} \Gamma_{\xi}^{(6)}\left(K_{1}^{\prime}, K_{2}^{\prime}, K_{3}^{\prime} ; K_{3}, K_{2}, K_{1}\right)= \\
& -\zeta \int_{K} \frac{\delta\left(\Omega_{K}-\xi\right)}{i \omega_{n}-\epsilon_{\mathbf{k}}+\mu-\Sigma_{\xi}(K)} \Gamma_{\xi}^{(8)}\left(K_{1}^{\prime}, K_{2}^{\prime}, K_{3}^{\prime}, K ; K, K_{3}, K_{2}, K_{1}\right) \\
& +3 \int_{K} \frac{\delta\left(\Omega_{K}-\xi\right) G_{\xi, \xi_{0}}\left(K^{\prime}\right)}{i \omega_{n}-\epsilon_{\mathbf{k}}+\mu-\Sigma_{\xi}(K)} \\
& \times\left\{\mathcal{A}_{\left(1^{\prime}, 2^{\prime}\right), 3^{\prime}}\left[\Gamma_{\xi}^{(4)}\left(K_{1}^{\prime}, K_{2}^{\prime} ; K^{\prime}, K\right) \Gamma_{\xi}^{(6)}\left(K, K^{\prime}, K_{3}^{\prime} ; K_{3}, K_{2}, K_{1}\right)\right]_{K^{\prime}=K_{1}^{\prime}+K_{2}^{\prime}-K}\right. \\
& \left.+\mathcal{A}_{1,(3,2)}\left[\Gamma_{\xi}^{(6)}\left(K_{1}^{\prime}, K_{2}^{\prime}, K_{3}^{\prime} ; K^{\prime}, K, K_{1}\right) \Gamma_{\xi}^{(4)}\left(K, K^{\prime} ; K_{3}, K_{2}\right)\right]_{K^{\prime}=K_{2}+K_{3}-K}\right\} \\
& +9 \zeta \int_{K}\left[\frac{\delta\left(\Omega_{K}-\xi\right) G_{\xi, \xi_{0}}\left(K^{\prime}\right)}{i \omega_{n}-\epsilon_{\mathbf{k}}+\mu-\Sigma_{\xi}(K)}+\frac{G_{\xi, \xi_{0}}(K) \delta\left(\Omega_{K^{\prime}}-\xi\right)}{i \omega_{n^{\prime}}-\epsilon_{\mathbf{k}^{\prime}}+\mu-\Sigma_{\xi}\left(K^{\prime}\right)}\right] \\
& \times \mathcal{A}_{\left(1^{\prime}, 2^{\prime}\right), 3^{\prime}} \mathcal{A}_{(2,1), 3}\left[\Gamma_{\xi}^{(6)}\left(K_{1}^{\prime}, K_{2}^{\prime}, K^{\prime} ; K, K_{2}, K_{1}\right) \Gamma_{\xi}^{(4)}\left(K_{3}^{\prime}, K ; K^{\prime}, K_{3}\right)\right]_{K^{\prime}=K+K_{3}^{\prime}-K_{3}} \\
& +9 \int_{K}\left[\frac{\delta\left(\Omega_{K}-\xi\right) G_{\xi, \xi_{0}}\left(K^{\prime}\right) G_{\xi, \xi_{0}}\left(K^{\prime \prime}\right)}{i \omega_{n}-\epsilon_{\mathbf{k}}+\mu-\Sigma_{\xi}(K)}+\frac{G_{\xi, \xi_{0}}(K) \delta\left(\Omega_{K^{\prime}}-\xi\right) G_{\xi, \xi_{0}}\left(K^{\prime \prime}\right)}{i \omega_{n^{\prime}}-\epsilon_{\mathbf{k}^{\prime}}+\mu-\Sigma_{\xi}\left(K^{\prime}\right)}\right. \\
& \left.+\frac{G_{\xi, \xi_{0}}(K) G_{\xi, \xi_{0}}\left(K^{\prime}\right) \delta\left(\Omega_{K^{\prime \prime}}-\xi\right)}{i \omega_{n^{\prime \prime}}-\epsilon_{\mathbf{k}^{\prime \prime}}+\mu-\Sigma_{\xi}\left(K^{\prime \prime}\right)}\right] \mathcal{A}_{\left(1^{\prime}, 2^{\prime}\right), 3^{\prime}} \mathcal{A}_{3,(2,1)} \\
& \times\left[\Gamma_{\xi}^{(4)}\left(K_{1}^{\prime}, K_{2}^{\prime} ; K, K^{\prime}\right) \Gamma_{\xi}^{(4)}\left(K_{3}^{\prime}, K^{\prime} ; K^{\prime \prime}, K_{3}\right) \Gamma_{\xi}^{(4)}\left(K^{\prime \prime}, K^{\prime} ; K_{2}, K_{1}\right)\right]_{K^{\prime}=K_{1}+K_{2}+K_{3}-K_{3}^{\prime}-K}^{K^{\prime \prime}=K_{2}+K_{1}-K} \\
& -36 \zeta \int_{K} \frac{\delta\left(\Omega_{K}-\xi\right) G_{\xi, \xi_{0}}\left(K^{\prime}\right) G_{\xi, \xi_{0}}\left(K^{\prime \prime}\right)}{i \omega_{n}-\epsilon_{\mathbf{k}}+\mu-\Sigma_{\xi}(K)} \mathcal{A}_{1^{\prime}, 2^{\prime}, 3^{\prime}} \mathcal{A}_{1,2,3} \\
& \times\left[\Gamma_{\xi}^{(4)}\left(K_{1}^{\prime}, K^{\prime} ; K, K_{1}\right) \Gamma_{\xi}^{(4)}\left(K_{2}^{\prime}, K^{\prime \prime} ; K^{\prime}, K_{2}\right) \Gamma_{\xi}^{(4)}\left(K_{3}^{\prime}, K ; K^{\prime \prime}, K_{3}\right)\right]_{K^{\prime}=K_{2}^{\prime}+K_{3}^{\prime}+K-K_{3}-K_{2}}^{K^{\prime \prime}=K_{3}^{\prime}+K-K_{3}} .
\end{aligned}
$$


Here the anti-symmetrization operators $\mathcal{A}_{1,2,3}$ and $\mathcal{A}_{1,(2,3)}$ are defined as follows,

$$
\begin{aligned}
\mathcal{A}_{1,2,3} f(1,2,3)=\frac{1}{6}[ & f(1,2,3)+f(2,3,1)+f(3,1,2) \\
& -f(3,2,1)-f(2,1,3)-f(1,3,2)], \\
\mathcal{A}_{1,(2,3)} f(1,2,3)= & \mathcal{A}_{(2,3), 1} f(1,2,3)=\frac{1}{3}[f(1,2,3)-f(2,1,3)-f(3,2,1)] .
\end{aligned}
$$

Given a function $f(1,2,3)$ that is already antisymmetric with respect to the pair $(2,3)$, the function $\mathcal{A}_{1,(2,3)} f(1,2,3)$ is a totally antisymmetric function. Note that the combinatorial factors in front of the terms involving the combinations $\Gamma^{(4)} \Gamma^{(6)}$ and $\Gamma^{(4)} \Gamma^{(4)} \Gamma^{(4)}$ are precisely the same as in the corresponding flow equation of $\phi^{4}$-theory, see Eq.(4.20) of Ref. [16].

The rescaled version of the above flow equation is (setting now $\zeta=-1$ )

$$
\begin{aligned}
& \partial_{t} \tilde{\Gamma}_{t}^{(6)}\left(Q_{1}^{\prime}, Q_{2}^{\prime}, Q_{3}^{\prime} ; Q_{3}, Q_{2}, Q_{1}\right)= \\
& \left\{-1-\sum_{i=1}^{3}\left[\frac{\eta_{t}^{\hat{\mathbf{n}}_{i}^{\prime}}+\eta_{t}^{\hat{\mathbf{n}}_{i}}}{2}+Q_{i}^{\prime} \cdot \partial_{Q_{i}^{\prime}}+Q_{i} \cdot \partial_{Q_{i}}\right]\right\} \tilde{\Gamma}_{t}^{(6)}\left(Q_{1}^{\prime}, Q_{2}^{\prime}, Q_{3}^{\prime} ; Q_{3}, Q_{2}, Q_{1}\right) \\
& -\int_{Q} \dot{G}_{t}(Q) \tilde{\Gamma}_{t}^{(8)}\left(Q_{1}^{\prime}, Q_{2}^{\prime}, Q_{3}^{\prime}, Q ; Q, Q_{3}, Q_{2}, Q_{1}\right) \\
& -3 \int_{Q} \dot{G}_{t}(Q) \tilde{G}_{t}\left(Q^{\prime}\right)\left\{\mathcal{A}_{\left(1^{\prime}, 2^{\prime}\right), 3^{\prime}}\left[\tilde{\Gamma}_{t}^{(4)}\left(Q_{1}^{\prime}, Q_{2}^{\prime} ; Q^{\prime}, Q\right) \tilde{\Gamma}_{t}^{(6)}\left(Q, Q^{\prime}, Q_{3}^{\prime} ; Q_{3}, Q_{2}, Q_{1}\right)\right]_{K^{\prime}=K_{1}^{\prime}+K_{2}^{\prime}-K}\right. \\
& \left.\quad+\mathcal{A}_{1,(3,2)}\left[\tilde{\Gamma}_{t}^{(6)}\left(Q_{1}^{\prime}, Q_{2}^{\prime}, Q_{3}^{\prime} ; Q^{\prime}, Q, Q_{1}\right) \tilde{\Gamma}_{t}^{(4)}\left(Q, Q^{\prime} ; Q_{3}, Q_{2}\right)\right]_{K^{\prime}=K_{2}+K_{3}-K}\right\} \\
& +9 \int_{Q}\left[\dot{G}_{t}(Q) \tilde{G}_{t}\left(Q^{\prime}\right)+\tilde{G}_{t}(Q) \dot{G}_{t}\left(Q^{\prime}\right)\right] \\
& \quad \times \mathcal{A}_{\left(1^{\prime}, 2^{\prime}\right), 3^{\prime}} \mathcal{A}_{(2,1), 3}\left[\tilde{\Gamma}_{t}^{(6)}\left(Q_{1}^{\prime}, Q_{2}^{\prime}, Q^{\prime} ; Q, Q_{2}, Q_{1}\right) \tilde{\Gamma}_{t}^{(4)}\left(Q_{3}^{\prime}, Q ; Q^{\prime}, Q_{3}\right)\right]_{K^{\prime}=K+K_{3}^{\prime}-K_{3}} \\
& +9 \int_{Q}\left[\dot{G}_{t}(Q) \tilde{G}_{t}\left(Q^{\prime}\right) \tilde{G}_{t}\left(Q^{\prime \prime}\right)+\tilde{G}_{t}(Q) \dot{G}_{t}\left(Q^{\prime}\right) \tilde{G}_{t}\left(Q^{\prime \prime}\right)+\tilde{G}_{t}(Q) \tilde{G}_{t}\left(Q^{\prime}\right) \dot{G}_{t}\left(Q^{\prime \prime}\right)\right] \mathcal{A}_{\left(1^{\prime}, 2^{\prime}\right), 3^{\prime}} \mathcal{A}_{3,(2,1)} \\
& \quad \times\left[\tilde{\Gamma}_{t}^{(4)}\left(Q_{1}^{\prime}, Q_{2}^{\prime} ; Q, Q^{\prime}\right) \tilde{\Gamma}_{t}^{(4)}\left(Q_{3}^{\prime}, Q^{\prime} ; Q^{\prime \prime}, Q_{3}\right) \tilde{\Gamma}_{t}^{(4)}\left(Q^{\prime \prime}, Q^{\prime} ; Q_{2}, Q_{1}\right)\right]_{K^{\prime}=K_{1}+K_{2}+K_{3}-K_{3}^{\prime}-K}^{K^{\prime \prime}=K_{2}+K_{1}-K} \\
& -36 \int_{Q} \dot{G}_{t}(Q) \tilde{G}_{t}\left(Q^{\prime}\right) \tilde{G}_{t}\left(Q^{\prime \prime}\right) \mathcal{A}_{1^{\prime}, 2^{\prime}, 3^{\prime}} \mathcal{A}_{1,2,3} \\
& \quad \times\left[\tilde{\Gamma}_{t}^{(4)}\left(Q_{1}^{\prime}, Q^{\prime} ; Q, Q_{1}\right) \tilde{\Gamma}_{t}^{(4)}\left(Q_{2}^{\prime}, Q^{\prime \prime} ; Q^{\prime}, Q_{2}\right) \tilde{\Gamma}_{t}^{(4)}\left(Q_{3}^{\prime}, Q ; Q^{\prime \prime}, Q_{3}\right)\right]_{K^{\prime}=K_{2}^{\prime}+K_{3}^{\prime}+K-K_{3}-K_{2}}^{K^{\prime \prime}=K_{3}^{\prime}+K-K_{3}}
\end{aligned}
$$

[1] D. Zanchi and H. J. Schulz, Phys. Rev. B 54, 9509 (1996); Z. Phys. B 103, 339 (1997); Phys. Rev. B 61, 13609 (2000).

[2] C. J. Halboth, PhD-Thesis (Shaker-Verlag, Aachen, 1999).

[3] C. J. Halboth and W. Metzner, Phys. Rev. B 61, 4364 (2000); Phys. Rev. Lett. 85, 5162 (2000).

[4] C. Honerkamp, PhD-Thesis, (ETH Zürich, 2000, unpublished).

[5] C. Honerkamp, M. Salmhofer, N. Furukawa, and T. M. Rice, Phys. Rev. B 63, 45114 (2001); M. Salmhofer and C. Honerkamp, Prog. Theor. Phys. 105, 1, (2001); C. Honerkamp, cond-mat/0103172.

[6] F. J. Wegner and A. Houghton, Phys. Rev. A 8, 401 (1973).

[7] K. G. Wilson, Phys. Rev. Lett. 26, 548 (1972); K. G. Wilson and J. G. Kogut, Phys. Reports 12C, 75 (1974).

[8] S. K. Ma, Modern Theory of Critical Phenomena (Benjamin/Cummings, Reading, Massachusetts, 1976). For a recent review, see M. E. Fisher, Rev. Mod. Phys. 70, 653 (1998).

[9] T. L. Bell and K. G. Wilson, Phys. Rev. B 74, 3935 (1974). 
[10] J. F. Nicoll, T. S. Chang, and H. E. Stanley, Phys. Lett. A 57, 7 (1976); J. F. Nicoll and T. S. Chang, ibid. 62, 287 (1977); T. S. Chang, D. D. Vvedensky, and J. F. Nicoll, Phys. Rep. 217, 279 (1992).

[11] J. Polchinski, Nucl. Phys. B 231, 269 (1984).

[12] C. Wetterich, Phys. Lett. B 301, 90 (1993).

[13] T. R. Morris, Int. J. Mod. Phys. A 9, 2411 (1994).

[14] M. Salmhofer, Renormalization (Springer, Berlin, 1998).

[15] C. Bagnuls and C. Bervillier, hep-th/0002034.

[16] P. Kopietz, Nucl. Phys. B 595, 493 (2001).

[17] R. Shankar, Rev. Mod. Phys. 66, 129 (1994).

[18] J. Zinn-Justin, Quantum Field Theory and Critical Phenomena (Clarendon Press, Oxford, 1989); M. Le Ballac, Quantum and Statistical Field Theory (Clarendon Press, Oxford, 1991).

[19] J. M. Luttinger, Phys. Rev. 119, 1153 (1960).

[20] If $\mathbf{k}$ lies outside a closed Fermi surface (as shown in Fig.11), then the mapping $\mathbf{k} \rightarrow \mathbf{k}_{F}$ defined by $\mathbf{k}=\mathbf{k}_{F}+\hat{\mathbf{v}}_{F} p$ yields a unique $\mathbf{k}_{F}$ for each $\mathbf{k}$. On the other hand, if $\mathbf{k}$ lies inside a closed Fermi surface, then $\mathbf{k}=\mathbf{k}_{F}+\hat{\mathbf{v}}_{F} p$ may have more than one solution $\mathbf{k}_{F}$. In this case we choose the solution which minimizes $p=\left|\mathbf{k}-\mathbf{k}_{F}\right|$.

[21] K. B. Blagoev and K. S. Bedell, Phys. Rev. Lett. 79, 1106 (1997); M. Yamanaka, M. Oshikawa, and I. Affleck, ibid. 1110 (1997).

[22] J. W. Negele and H. Orland, Quantum many-particle systems, (Addison-Wesley, Redwood City, 1988).

[23] W. Metzner, C. Castellani, and C. Di Castro, Adv. Phys. 47, 317 (1998).

[24] J. Feldman, M. Salmhofer, and E. Trubowitz, J. Stat. Phys. 84, 1209 (1996); Commun. Pure Appl. Math. 51, 1133 (1998).

[25] U. Ellwanger, Z. Phys. C 62, 503 (1994).

[26] We introduce $\zeta=-1$ because then the bosonic version of the exact flow equations of Sec. II can be simply obtained by replacing $\zeta \rightarrow+1$.

[27] Although we use a different notation, our Eq.(2.16) is equivalent with the flow equation for the Legendre effective action given in Refs. [4, $\left[\right.$. We have adopted the same notation as Ref. [16] to emphasize the analogy with $\phi^{4}$-theory.

[28] G. Y. Chitov and D. Sénéchal, Phys. Rev. B 52, 13487 (1995); ibid. 57, 1444 (1998); N. Dupuis and G. Y. Chitov, ibid. 54, 3040 (1996).

[29] N. Dupuis, Eur. Phys. J. B 3, 315 (1998); Int. J. Mod. Phys. B 14, 379 (2000).

[30] C. Honerkamp and M. Salmhofer, cond-mat/0105218.

[31] K. Louis, J. V. Alvarez, and C. Gros, cond-mat/0103009.

[32] P. W. Anderson, Phys. Rev. Lett. 71, 1220 (1993).

[33] J. Comellas, Nucl. Phys. B 509, 602 (1998).

[34] J. Sólyom, Adv. Phys. 28, 201 (1979).

[35] P. Carta, Phys. Rev. B 62, 10687 (2000).

[36] J. Voit, Rep. Prog. Phys. 57, 977 (1994).

[37] T. Busche and P. Kopietz, unpublished.

[38] B. Binz, D. Baeriswyl, and B. Douçot, cond-mat/0104424.

[39] A. Ferraz, cond-mat/0104576

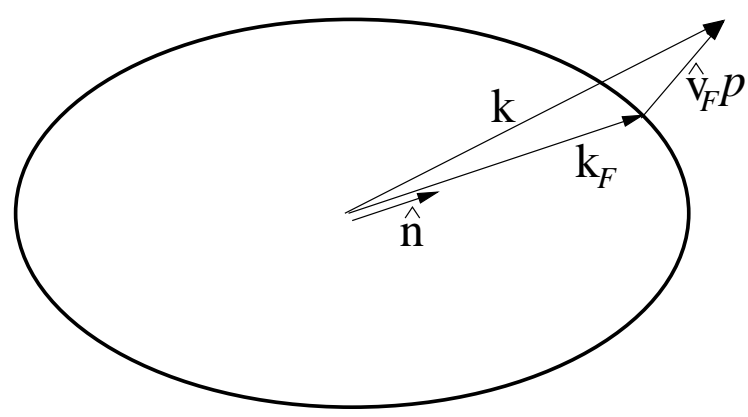

FIG. 1. An arbitrary vector $\mathbf{k}$ can be decomposed into a component $\mathbf{k}_{F}$ on the Fermi surface (here an ellipse) and a component $\hat{\mathbf{v}}_{F} p$ parallel to the local Fermi velocity, see Eq.(2.2). This construction defines $\mathbf{k}_{F}$ as a function of $\mathbf{k}$. 

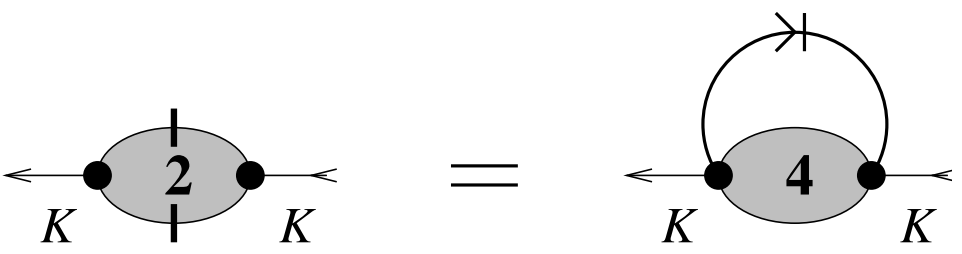

FIG. 2. Diagrammatic representation of the flow equation for the two-point vertex, see Eqs. 2.25) and (3.25). The left-hand side represents the derivative of the total irreducible vertex with respect to the flow parameter. The arrows represent the exact propagators, and an arrow with an extra slash represents the derivative of the cutoff function with respect to the flow parameter.
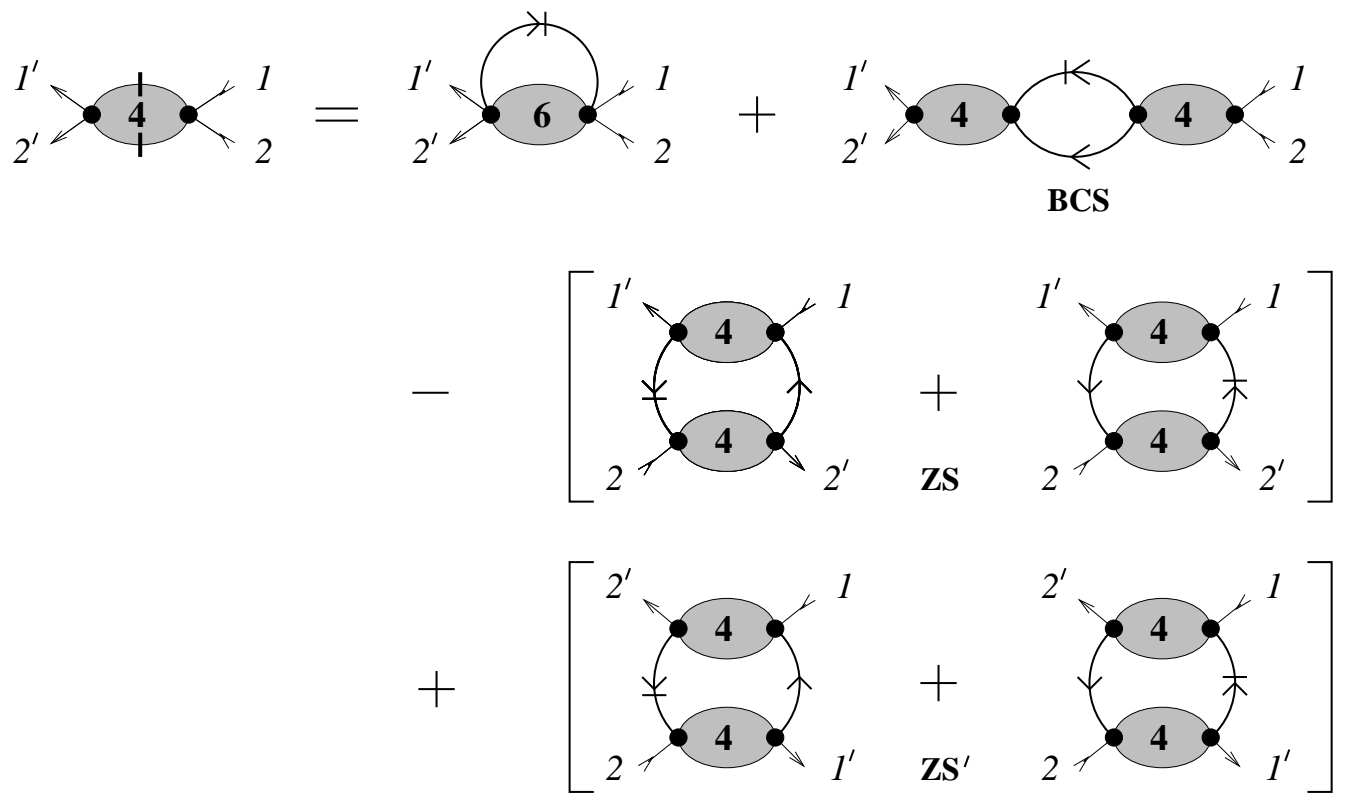

FIG. 3. Diagrammatic representation of the flow equation for the four-point vertex, see Eqs.(2.26) and (3.28). 


$$
\text { (1) }
$$

FIG. 4. Diagrammatic representation of the flow equation for the six-point vertex, see Eqs. A.1) and (A.4). 